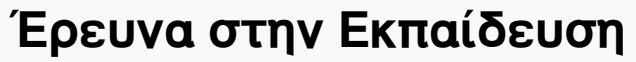

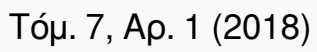

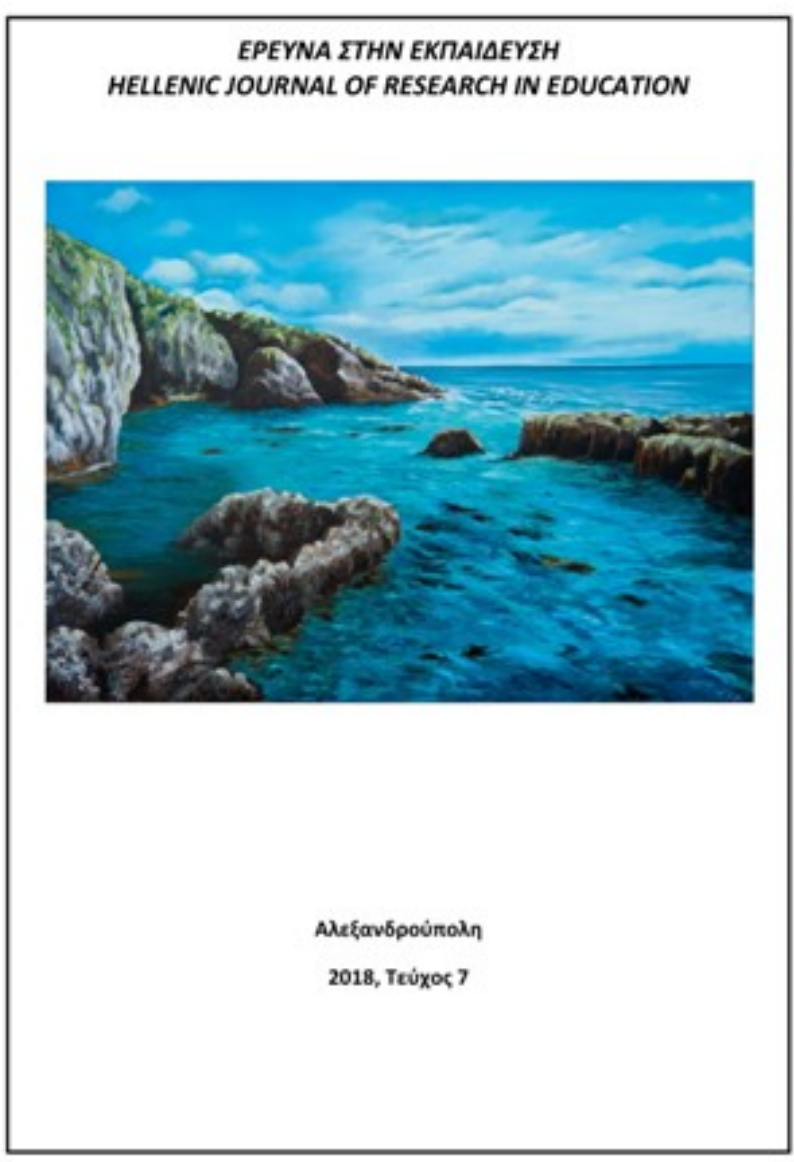

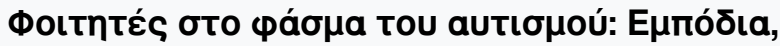

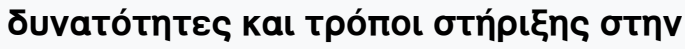

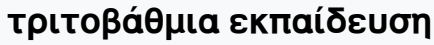

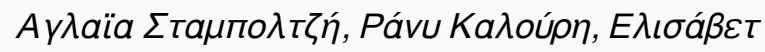
Tбítбou

doi: $\underline{10.12681 / \text { hjre. } 17413}$

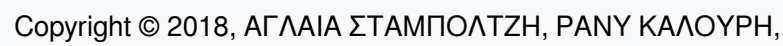

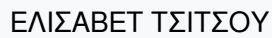

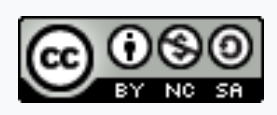

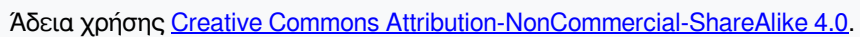

\section{Bıß入ıорачıкń avaчopá:}

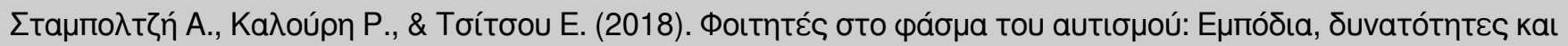

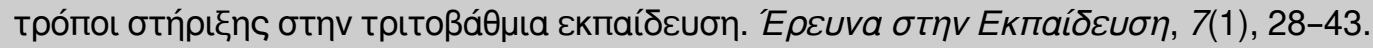

https://doi.org/10.12681/hjre.17413 


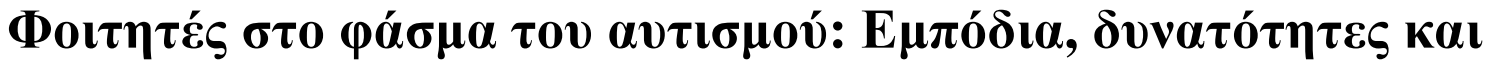

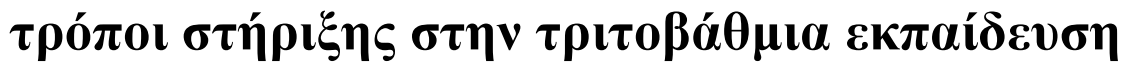

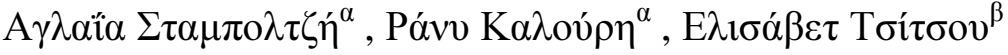

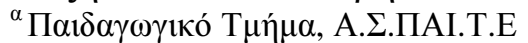

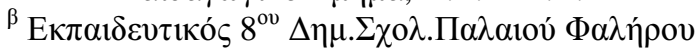

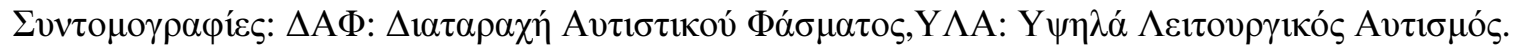

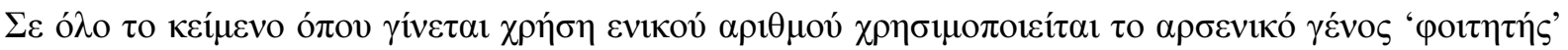

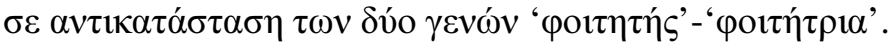

\section{Пврі́ $\eta \psi \eta$}

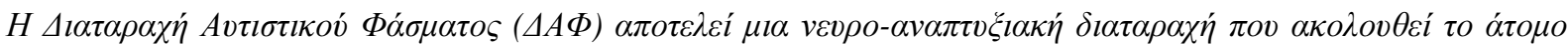

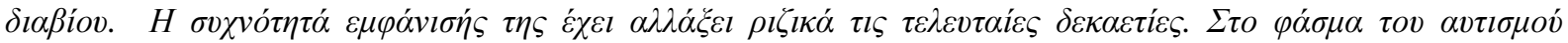

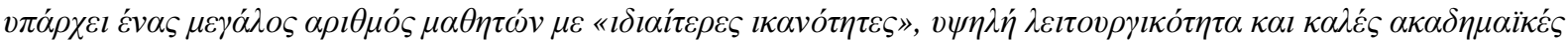

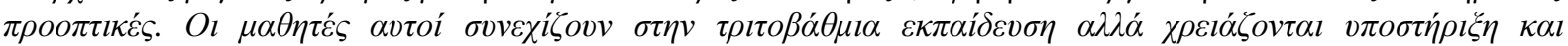

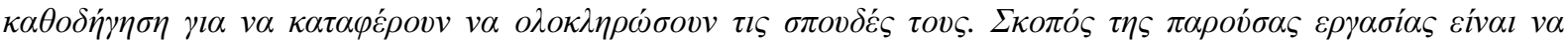

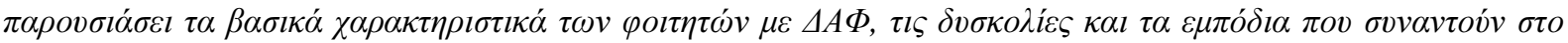

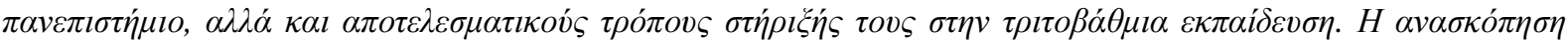

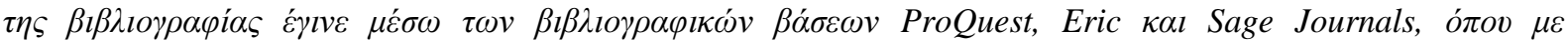

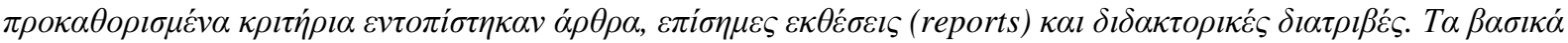

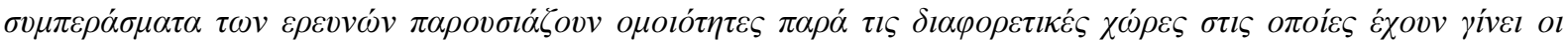

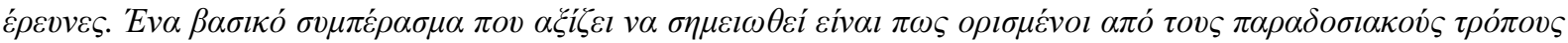

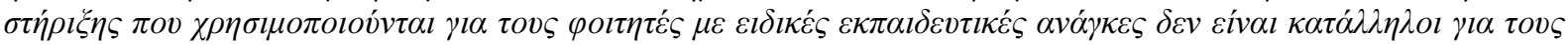

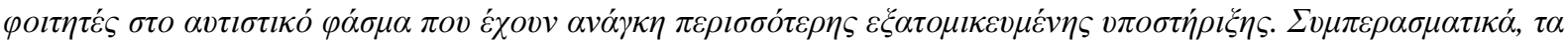

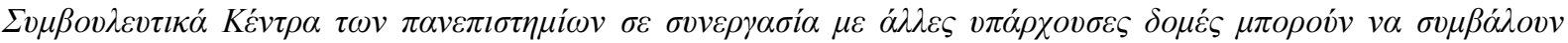

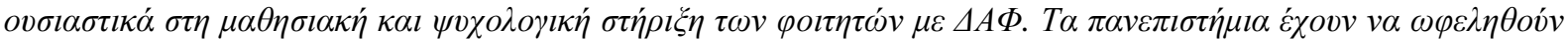

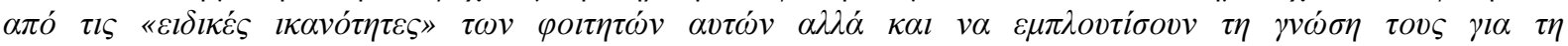

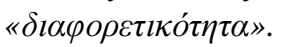

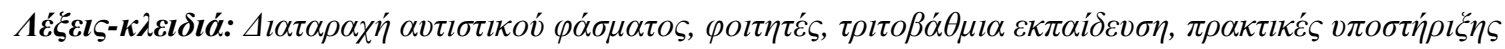

\begin{abstract}
Autism Spectrum Disorder (ASD) is a lifelong neurodevelopmental condition. The incidence of ASD is rising impressively during the last decades. Within the autism spectrum, there is a large number of students with "special abilities", above average or high intelligence and good academic prospects. A large number of these students moves to higher education, in order to pursue their personal and academic goals. The purpose of this paper is to present the basic characteristics of university students with ASD, the difficulties and obstacles they encounter at university, as well as effective ways of supporting them in higher education. The literature review was conducted through the electronic databases ProQuest, Eric and Sage Journals where specific inclusion criteria had been set for articles, reports and theses. The main outcomes of the studies have several similarities irrespective of the country of origin of the studies. Students with ASD meet emotional, practical, social, communicative and sensory challenges in higher education. One key conclusion is that some of the traditional support methods used for students with special educational needs are not suitable for students with autism who needs personalized support based on their unique needs. Personnel of counselling or disability centres should offer appropriate accommodations to support academically and emotionally students on the autistic spectrum, taking into account the context of the institution and the large variability between students with ASD. Finally,
\end{abstract}

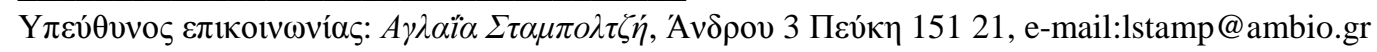

URL: http://ejournals.epublishing.ekt.gr/index.php/hjre/index 
universities have to benefit from the "special abilities" of these students and also enrich their knowledge of "diversity".

Key words: Autism spectrum disorder, students, higher education, supportive practices and accommodations

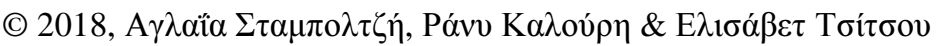

A $\delta \varepsilon 1 \alpha$ CC-BY-SA 4.0

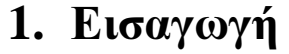

Oı

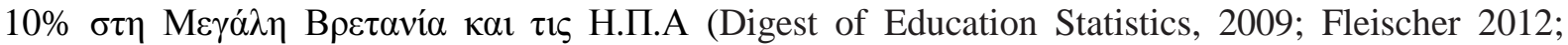

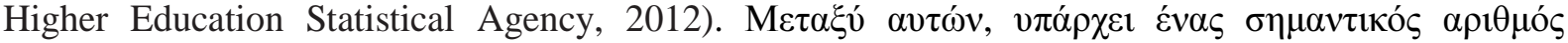

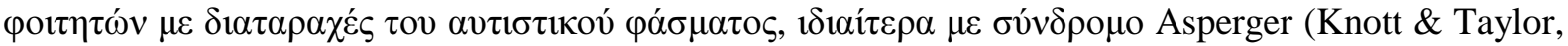

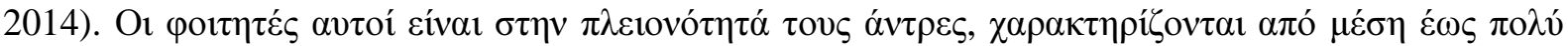

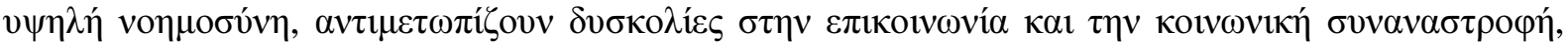

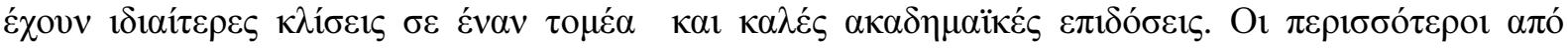

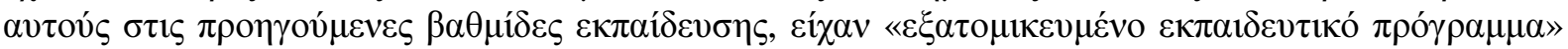

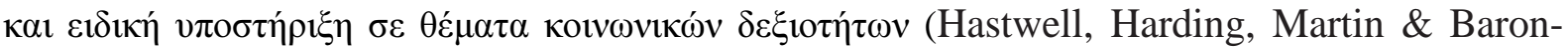

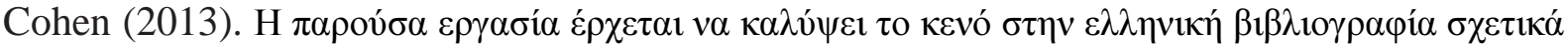

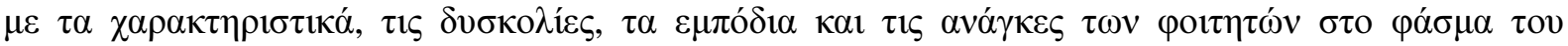

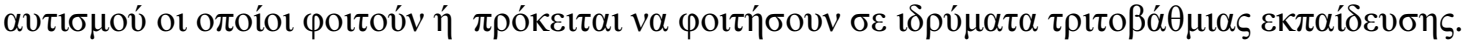

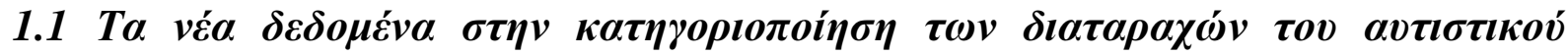 $\varphi \dot{\alpha} \sigma \mu \alpha \tau \sigma \varsigma$}

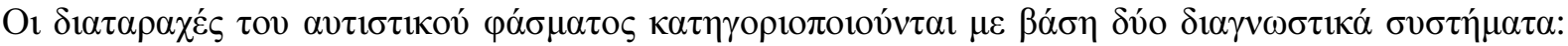

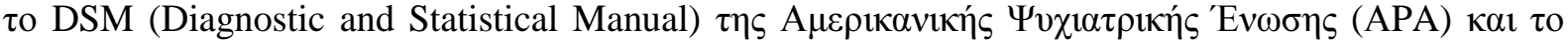

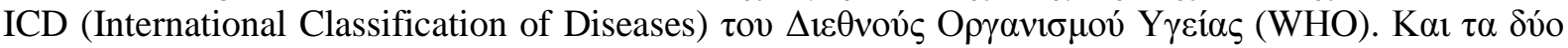

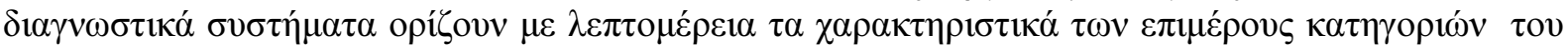

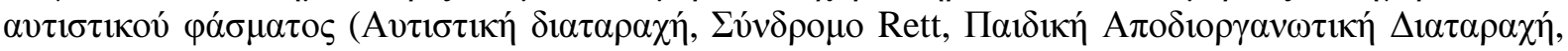
$\Sigma v ́ v \delta \rho o \mu o ~ A s p e r g e r \kappa . \lambda \pi$.) (Tsai, 2003).

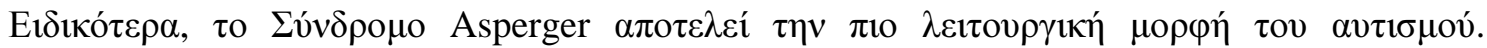

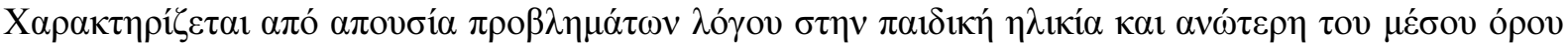

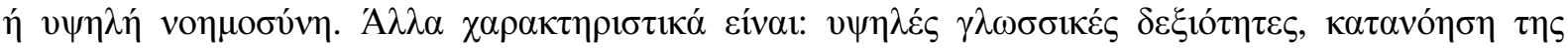

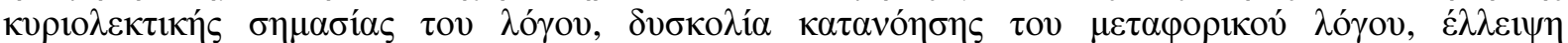

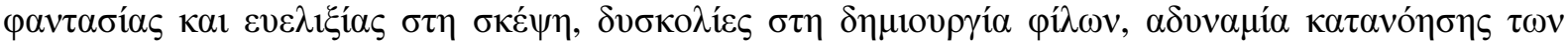

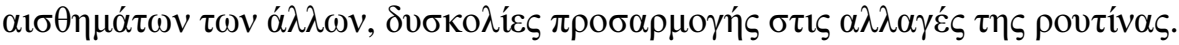

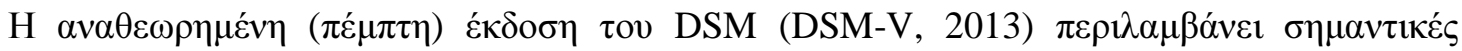

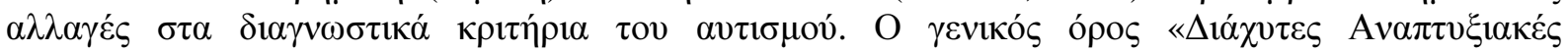

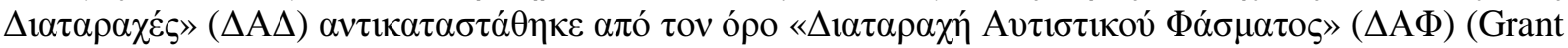
$\&$ Nozyce, 2013).

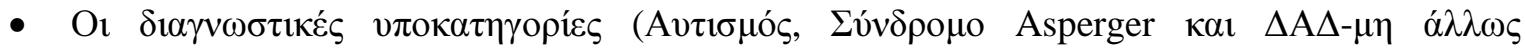
$\pi \rho о \sigma \delta 10 \rho 1 \zeta o ́ \mu \varepsilon v \eta) \alpha \pi \alpha \lambda \varepsilon i ́(\varphi \theta \eta \kappa \alpha v$.

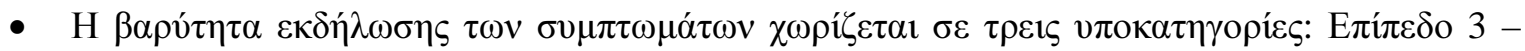

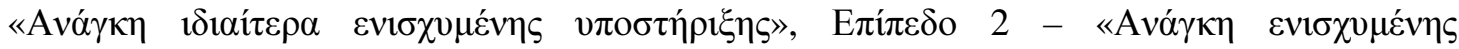

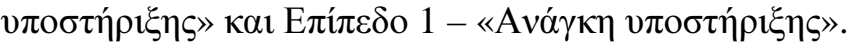

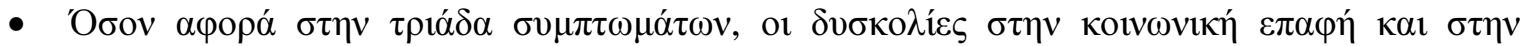

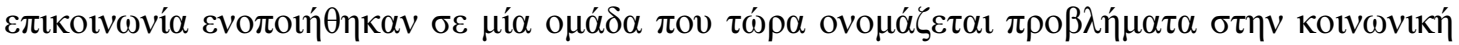

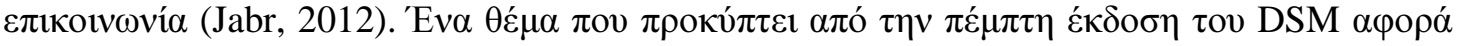




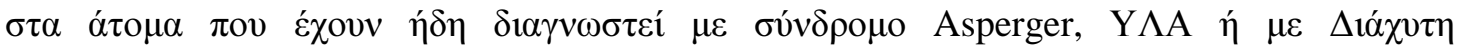

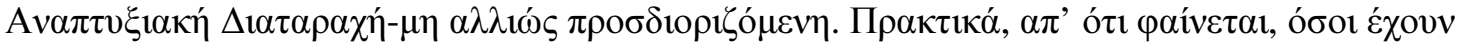

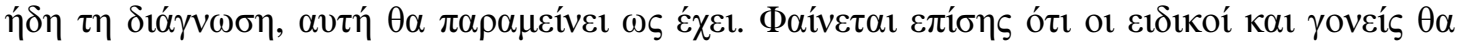

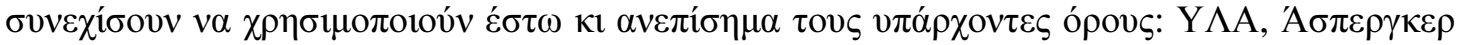

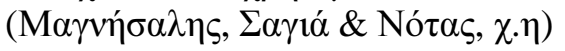

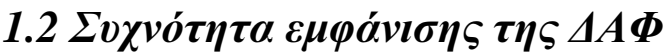

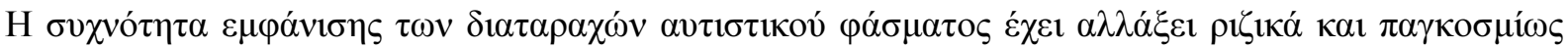

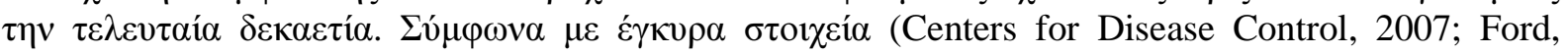

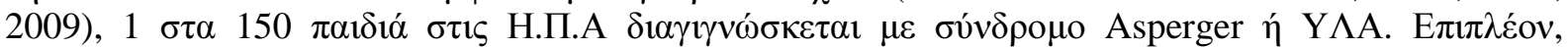

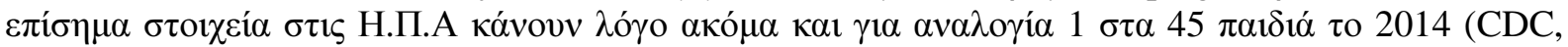

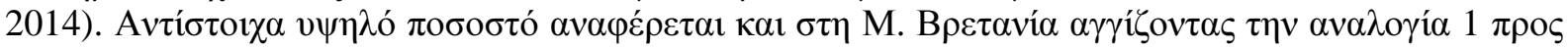

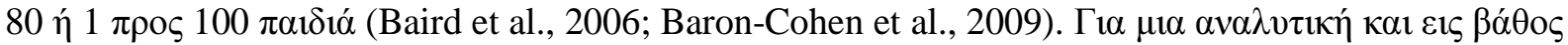

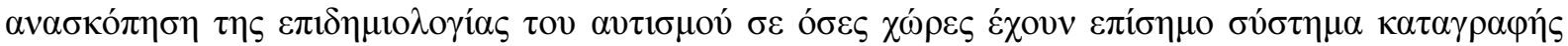

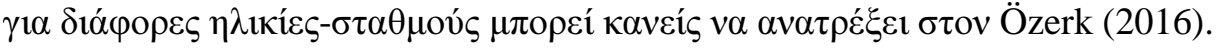

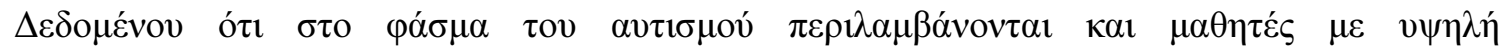

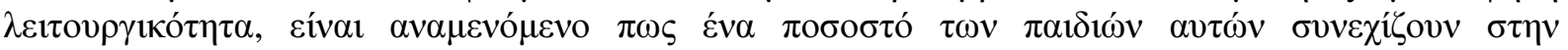

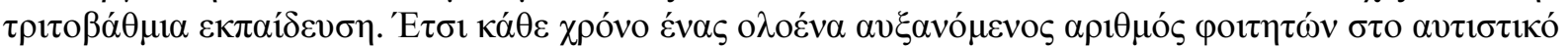

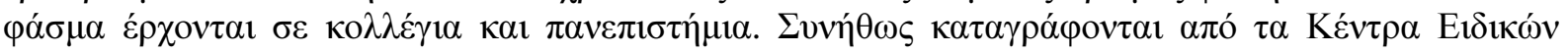

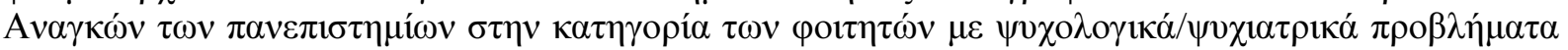

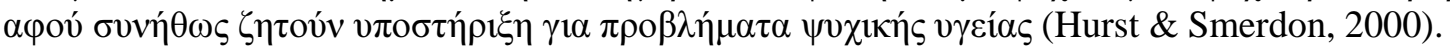

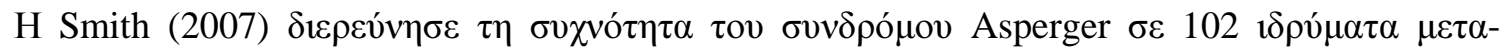

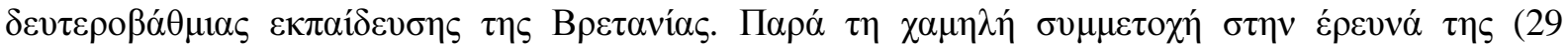

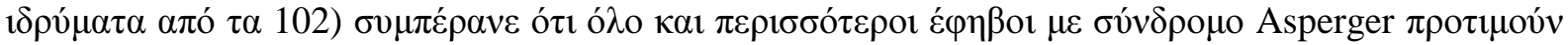

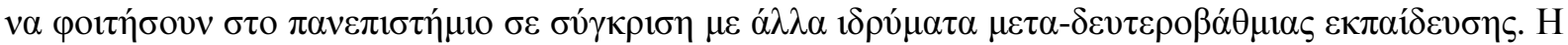

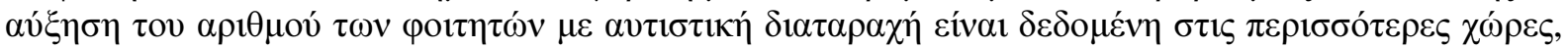

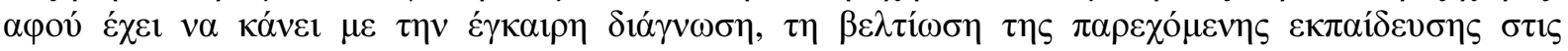

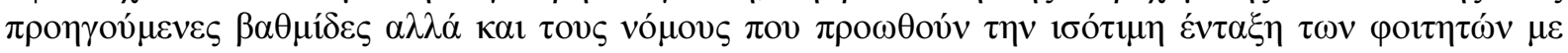

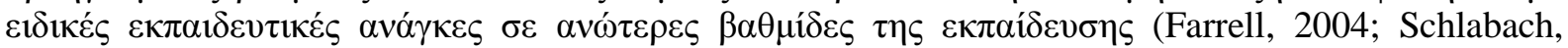
2008).

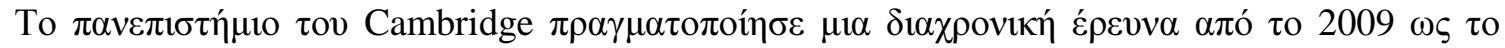

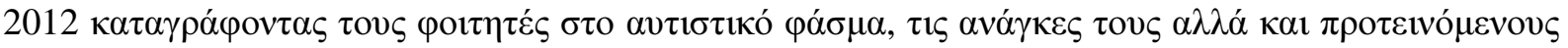

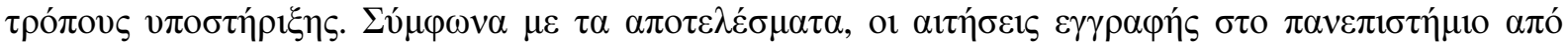

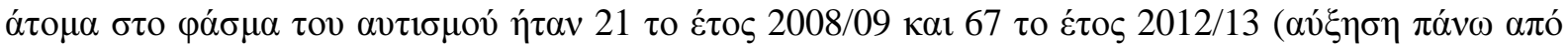

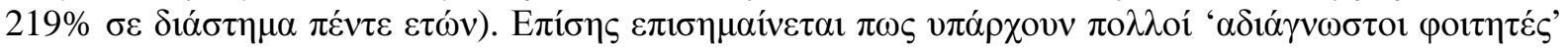

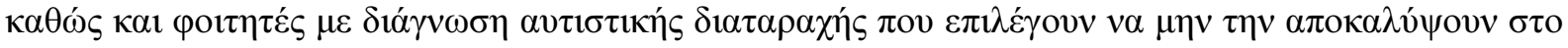

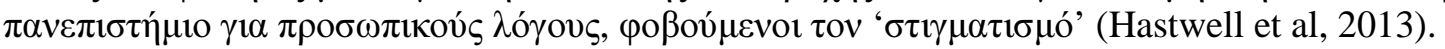

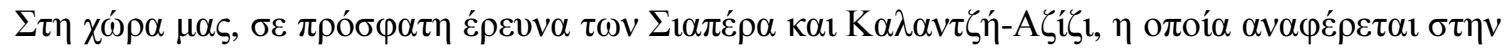

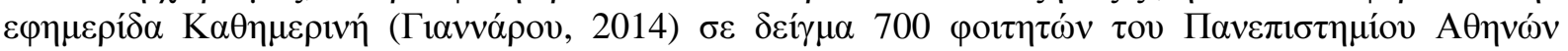

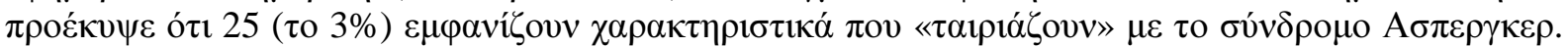

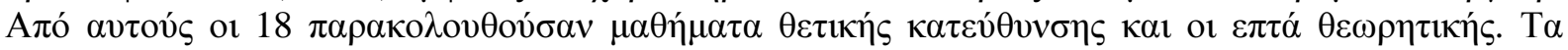

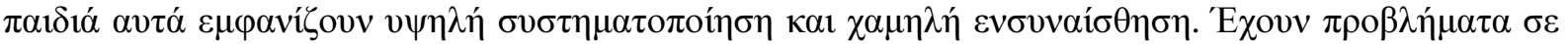

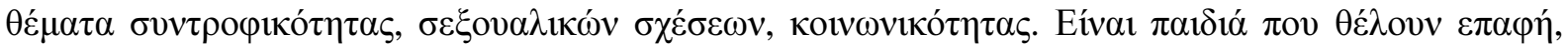

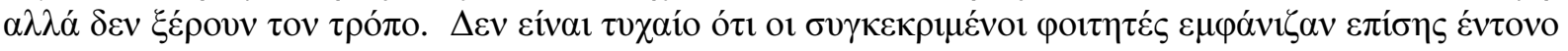

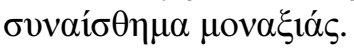

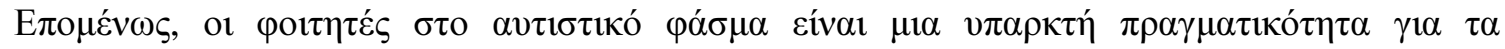

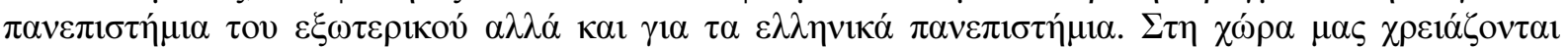

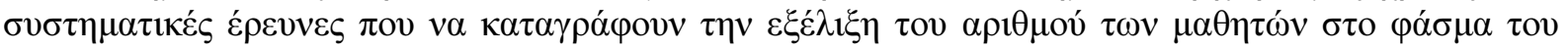




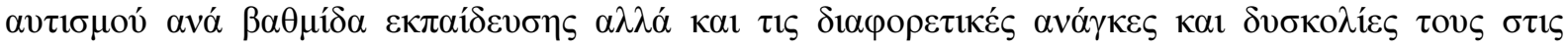

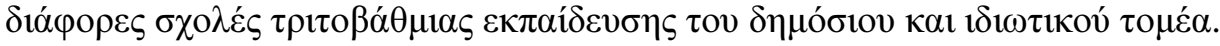

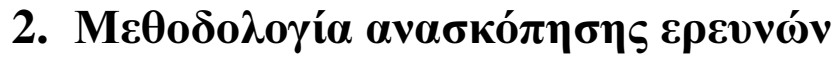

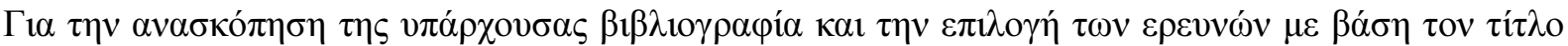

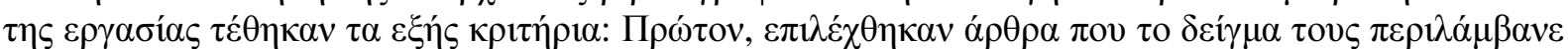

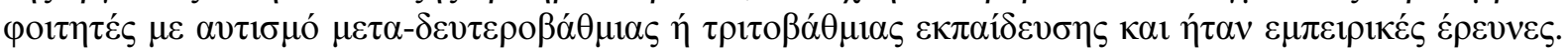

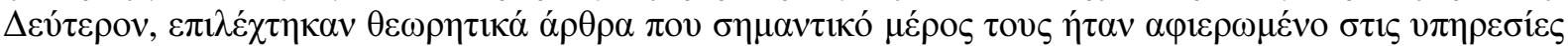

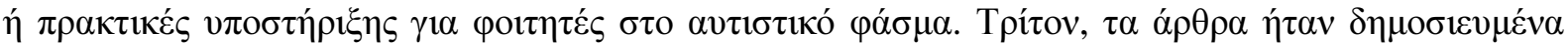

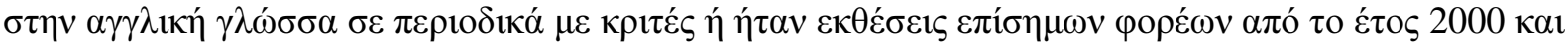
$\mu \varepsilon \tau$ ฉ́.

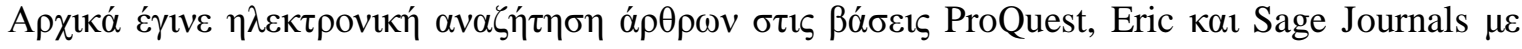

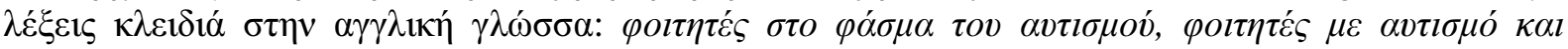

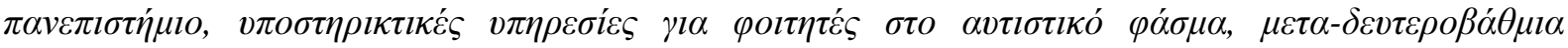

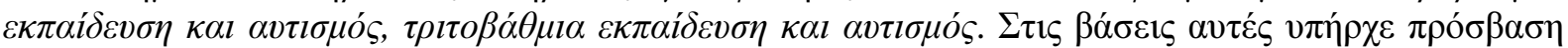

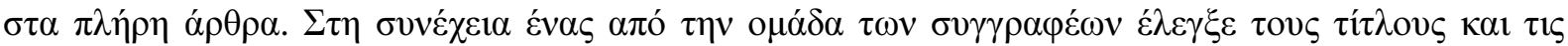

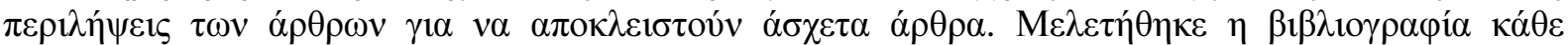

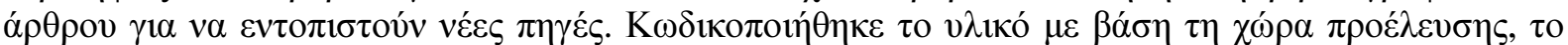

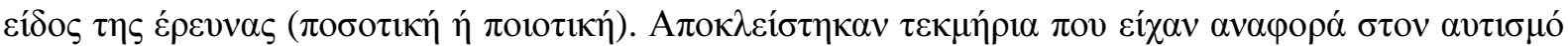

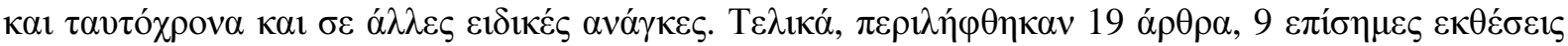

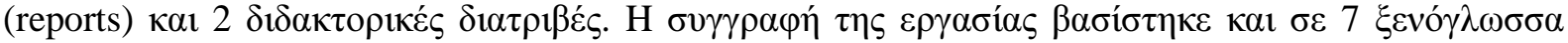

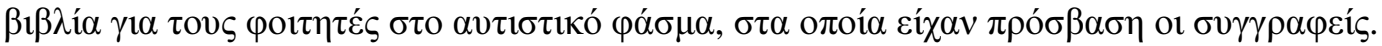

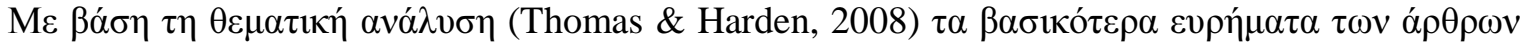

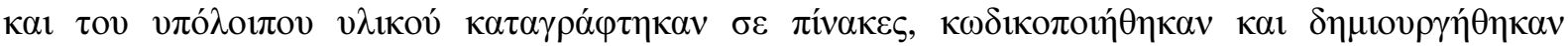

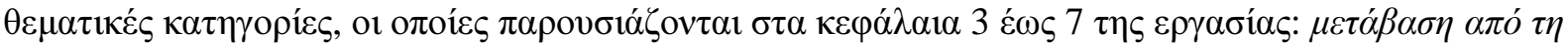

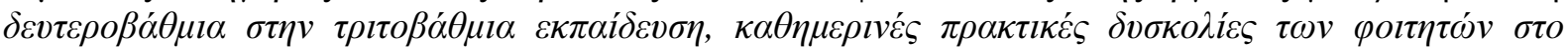

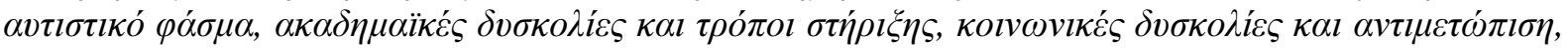

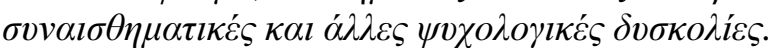

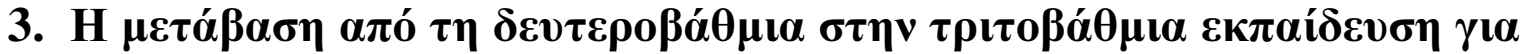

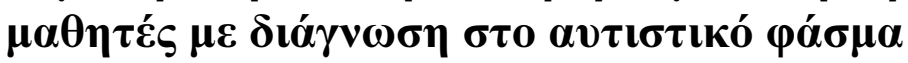

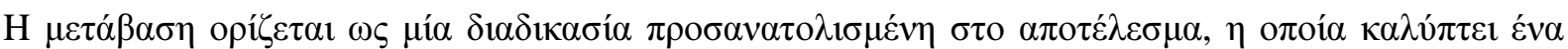

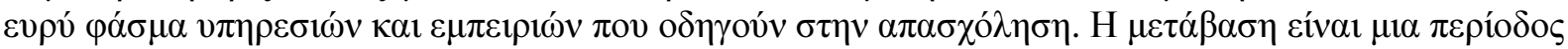

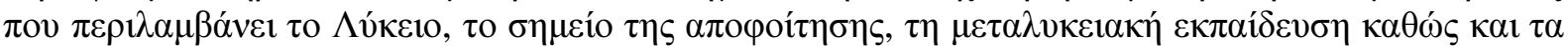

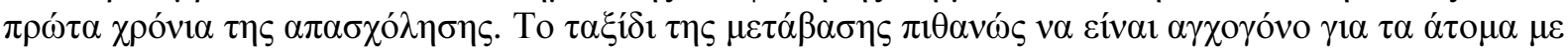

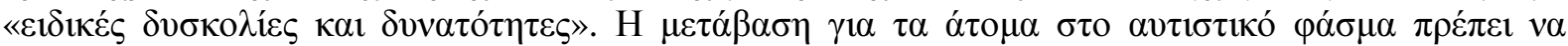

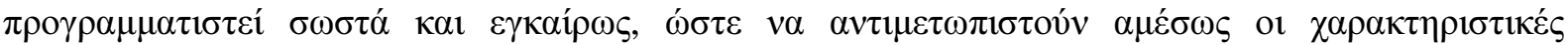

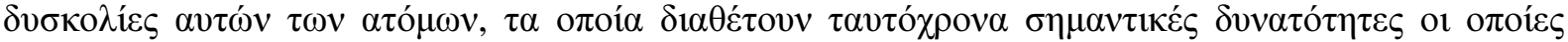

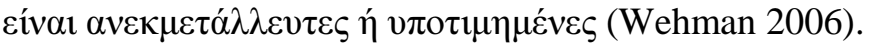

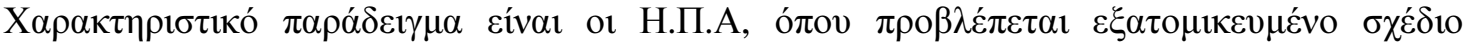

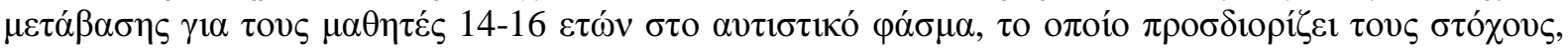

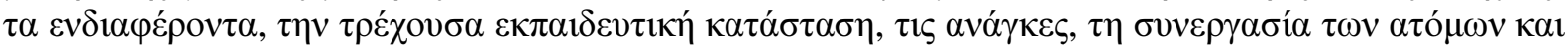

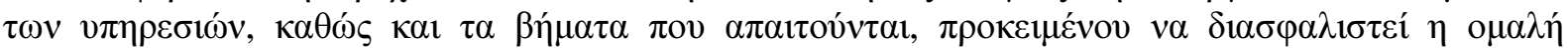

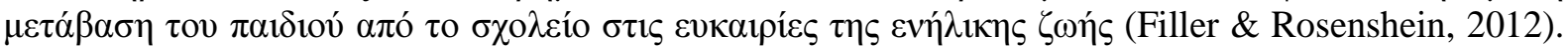




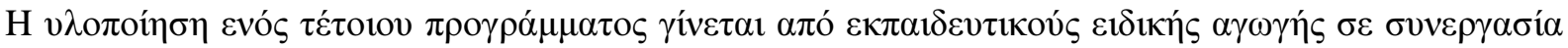

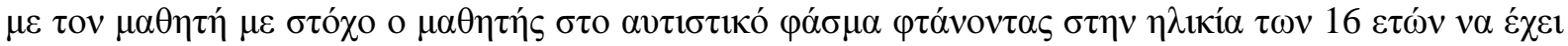

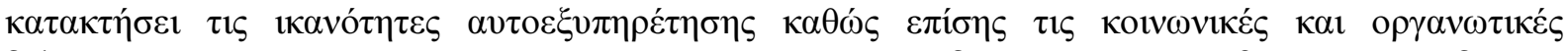

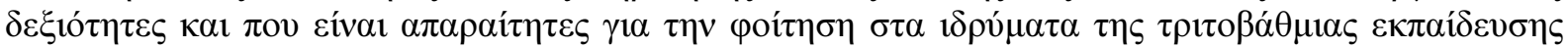
(Lawrence, Alleckson \& Bjorklund, 2010).

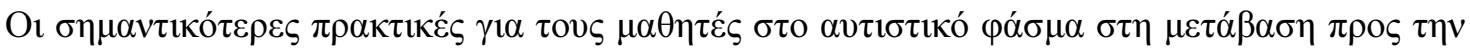

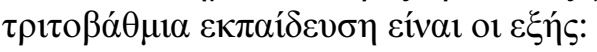

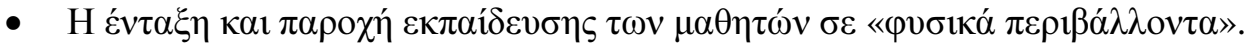

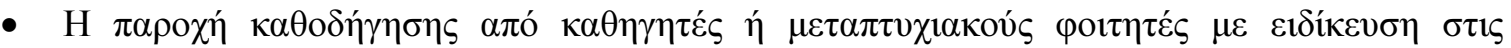

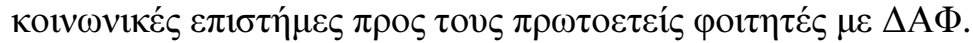

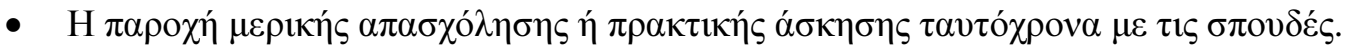

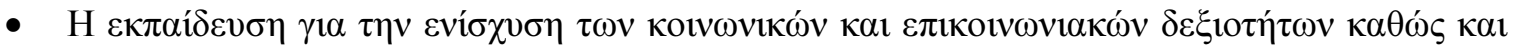

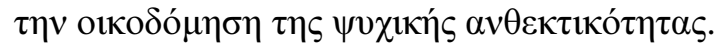

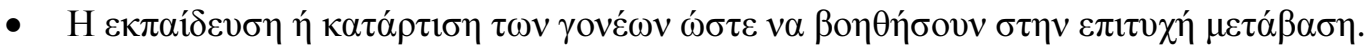

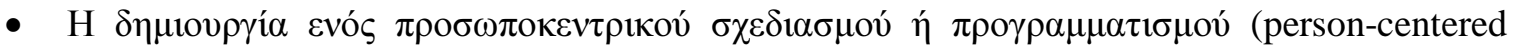

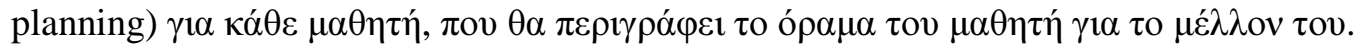

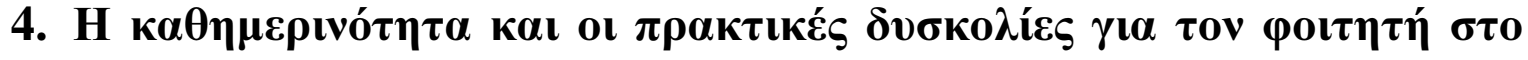

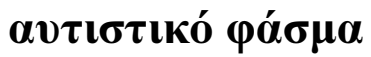

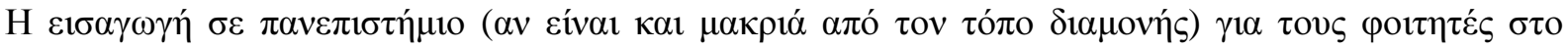

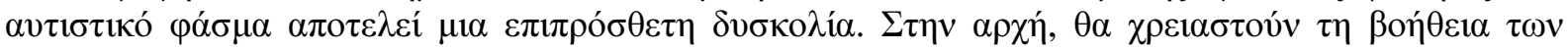

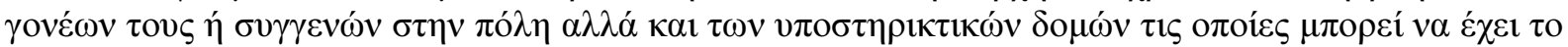

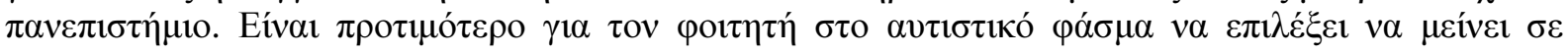

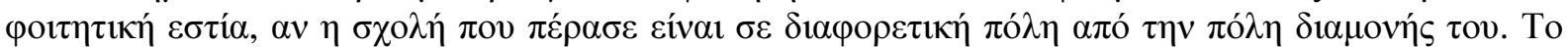

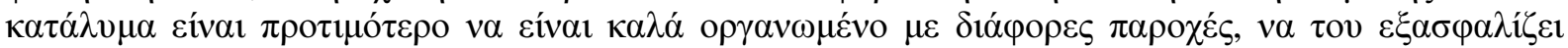

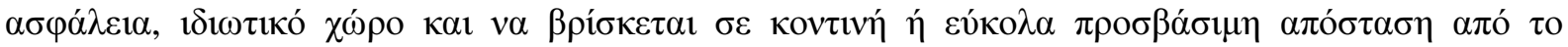

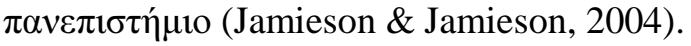

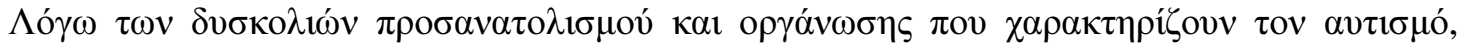

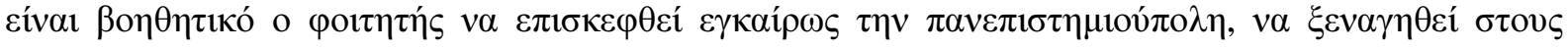

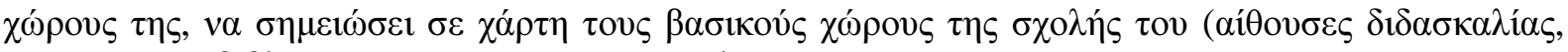

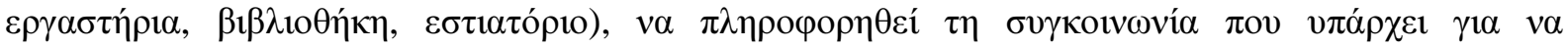

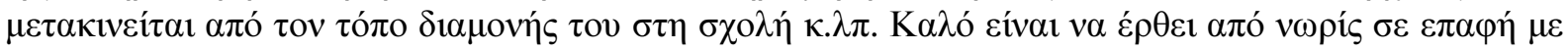

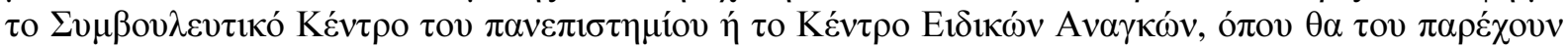

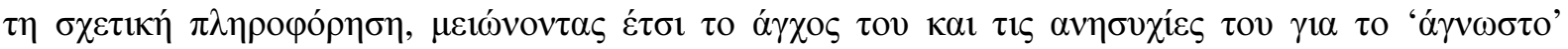

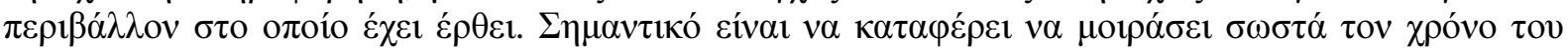

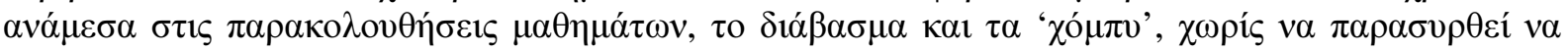

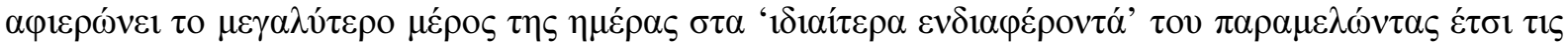

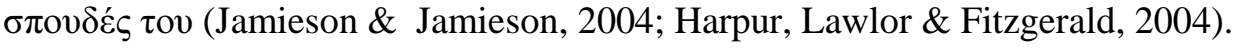

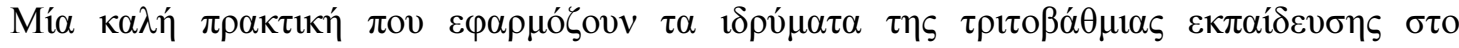

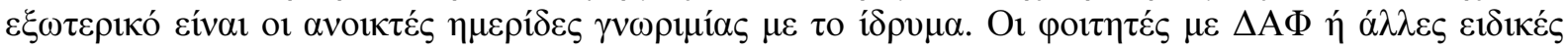

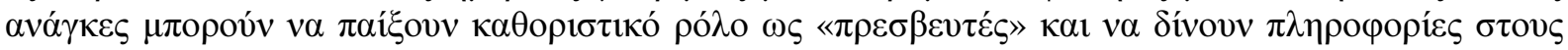

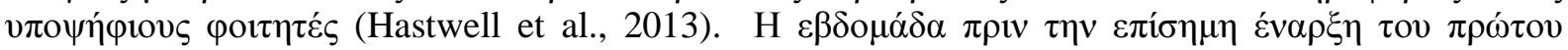

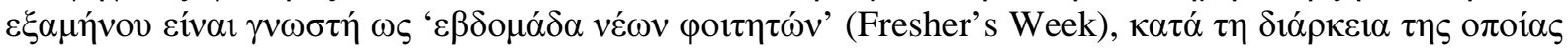

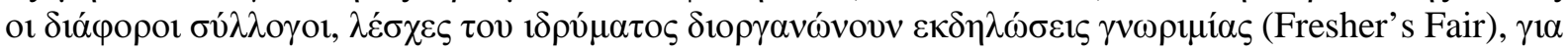

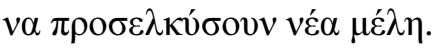

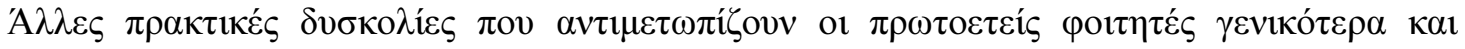

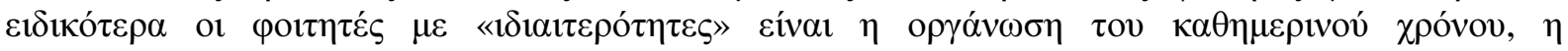




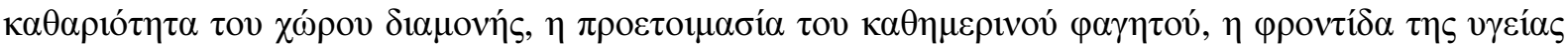

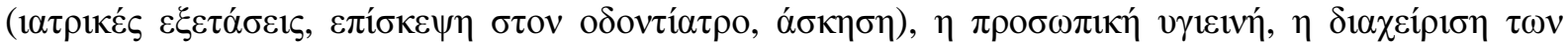

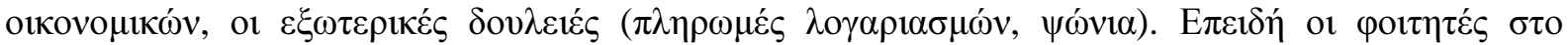

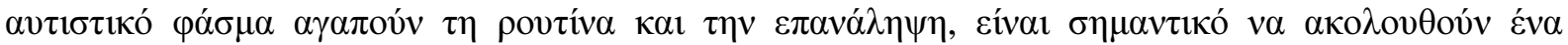

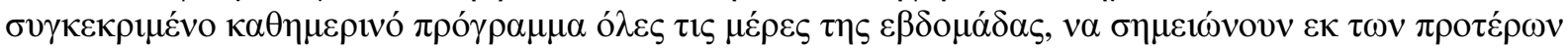

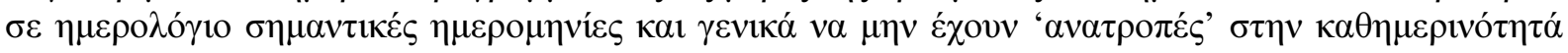

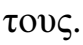

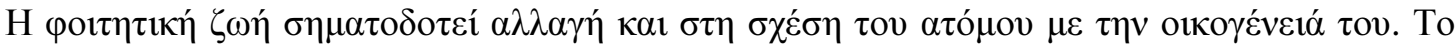

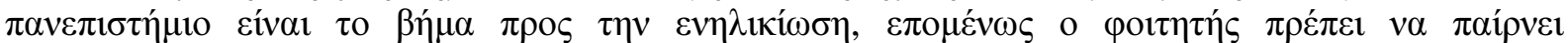

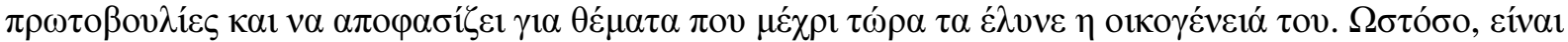

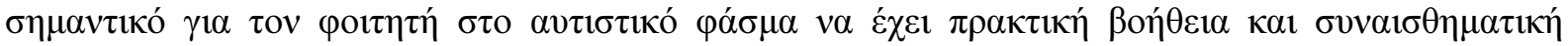

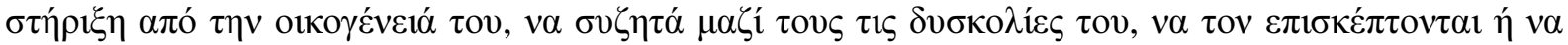

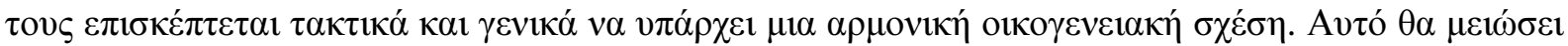

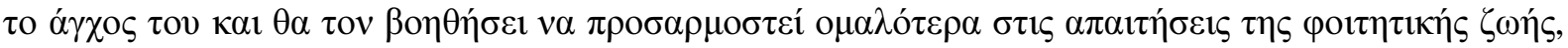

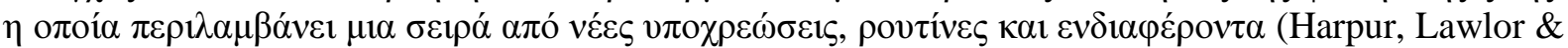
Fitzgerald, 2004).

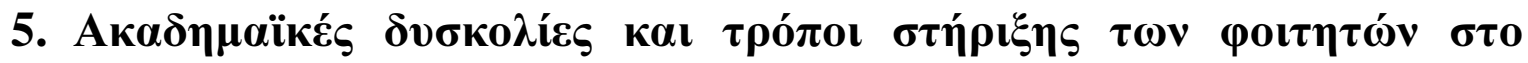

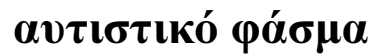

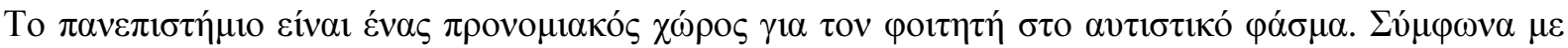

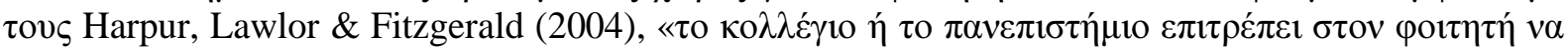

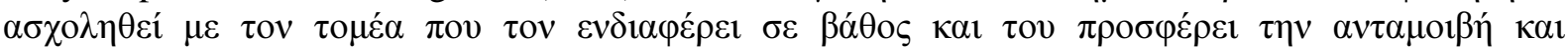

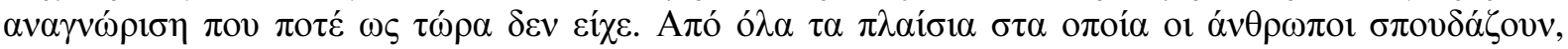

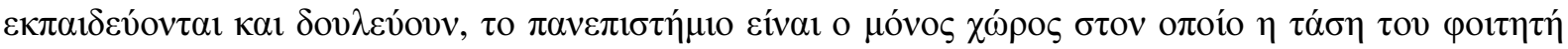

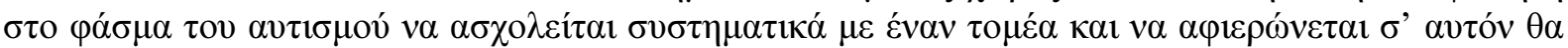

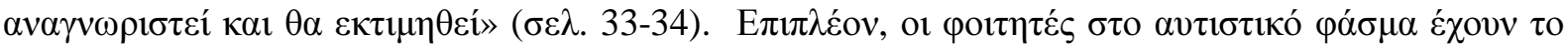

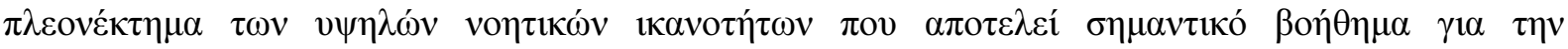

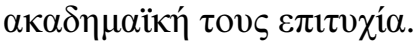

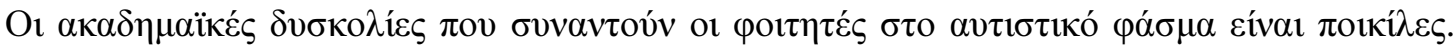

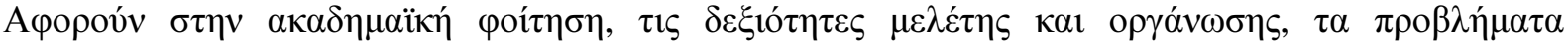

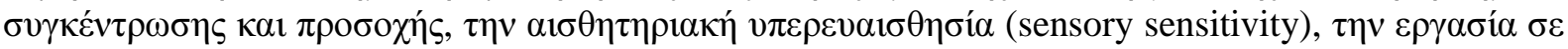

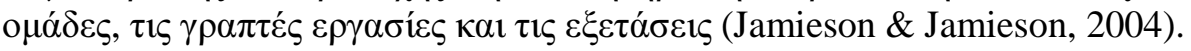

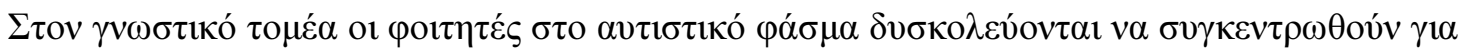

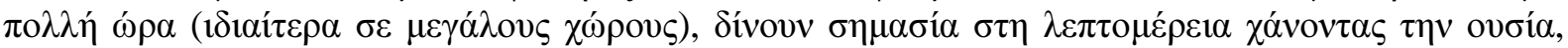

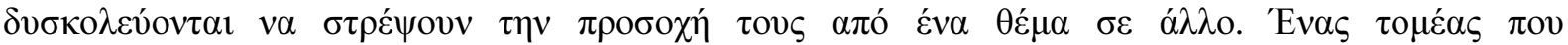

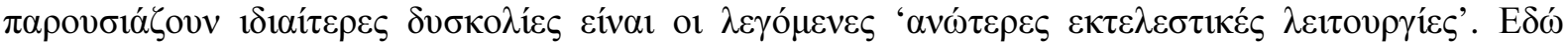

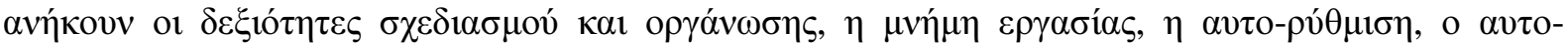

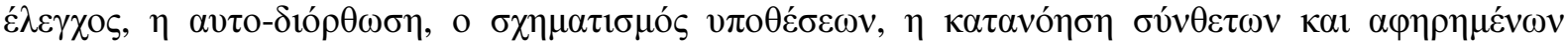

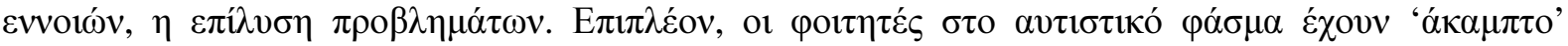

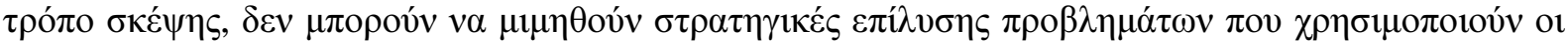

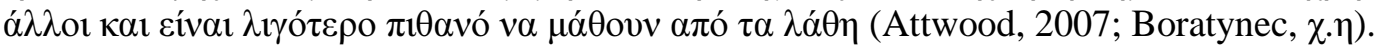

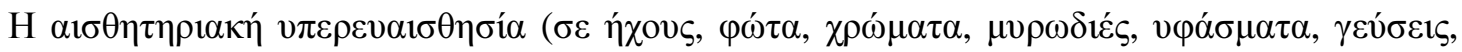

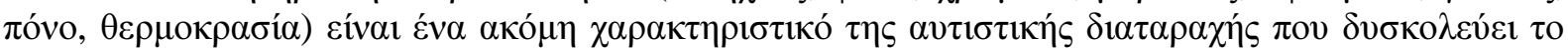

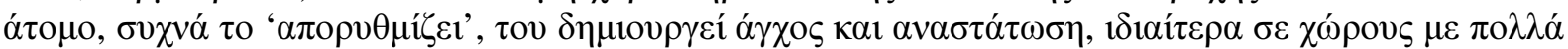

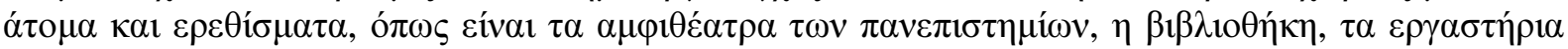

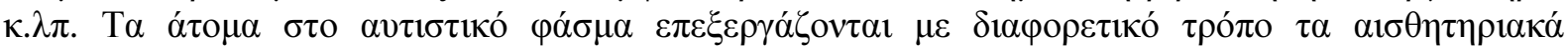




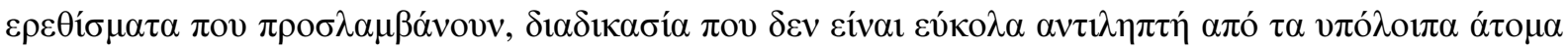
(Harrison \& Hare, 2004).

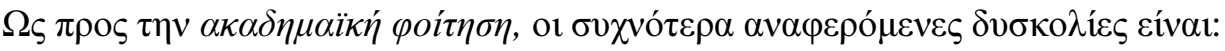

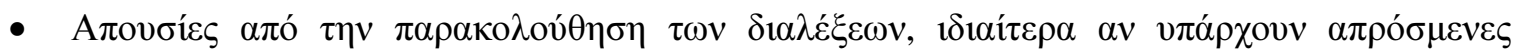
$\alpha \lambda \lambda \alpha \gamma \varepsilon \dot{\varsigma} \varsigma \tau \tau$ $\sigma \rho o ́ \gamma \rho \alpha \mu \mu \alpha$

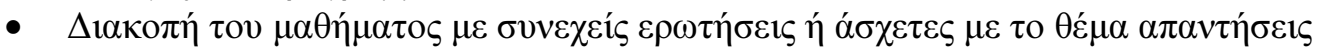

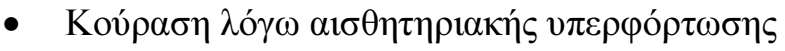

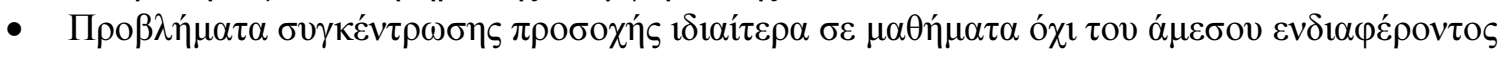

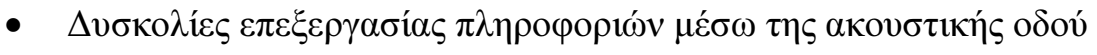

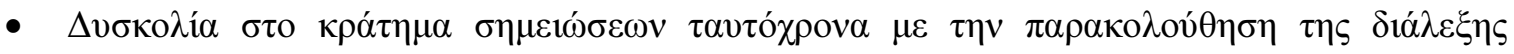
(Jamieson \& Jamieson, 2004)

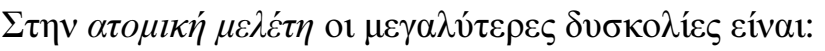

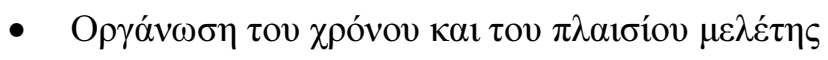

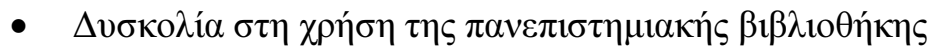

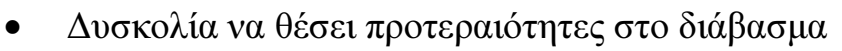

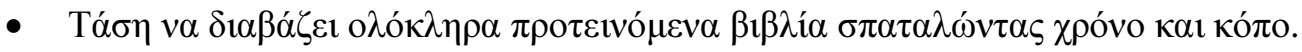

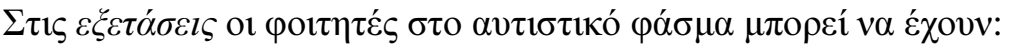

- $\Delta v \sigma \kappa o \lambda i ́ \varepsilon \varsigma \kappa \alpha \tau \alpha v o ́ \eta \sigma \eta \varsigma \tau \omega v \varepsilon \kappa \varphi \omega v \eta ́ \sigma \varepsilon \omega v-\varepsilon \rho \omega \tau \eta ́ \sigma \varepsilon \omega v$

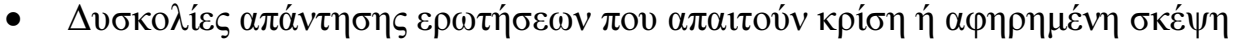

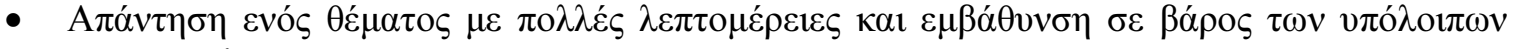

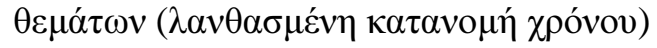

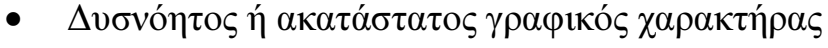

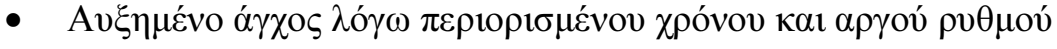

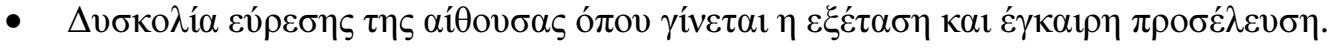

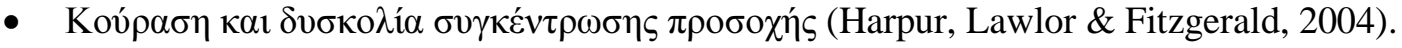

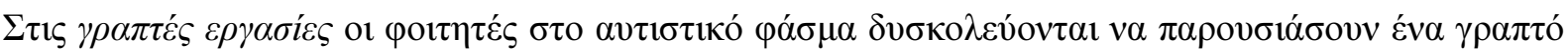

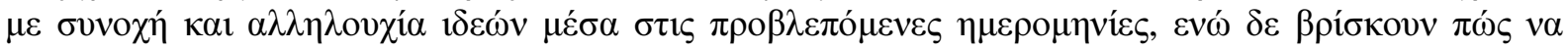

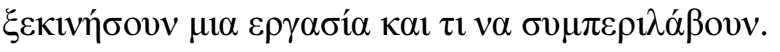

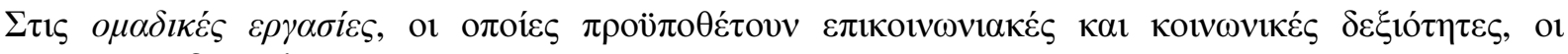

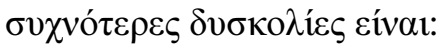

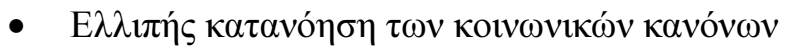

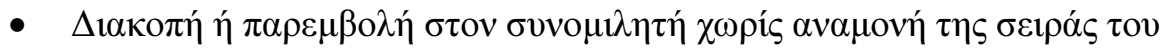

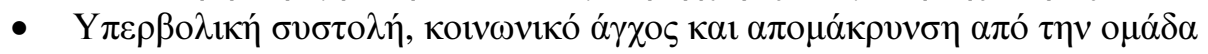

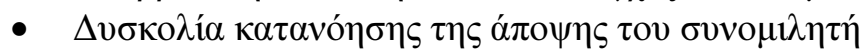

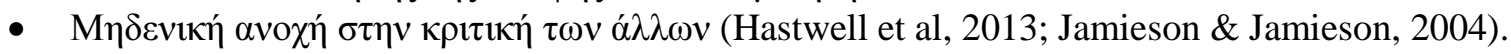

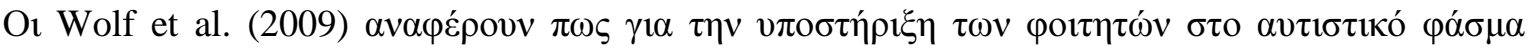

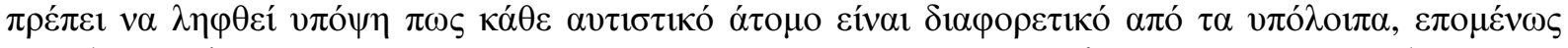

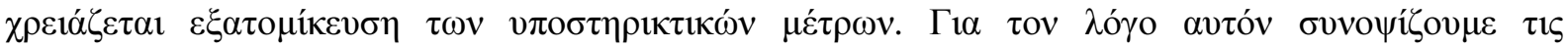

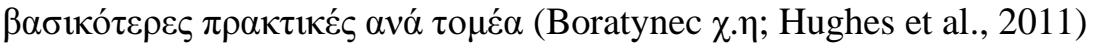

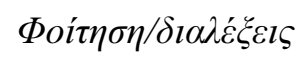

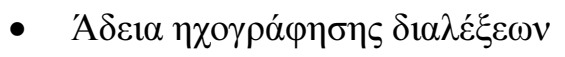




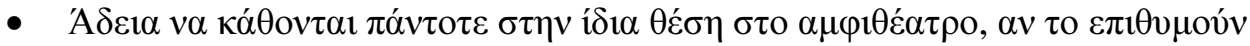

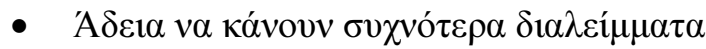

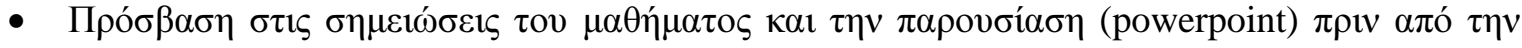
$\eta \mu \varepsilon ́ \rho \alpha \tau o v \mu \alpha \theta \eta \dot{\mu} \mu \alpha \tau \varsigma$

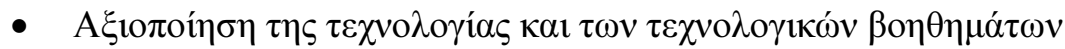

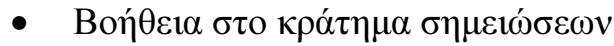

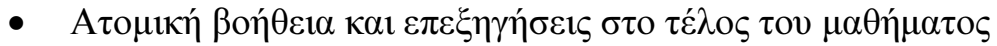

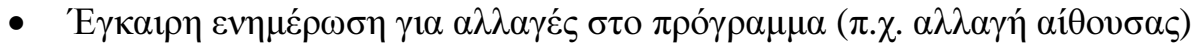

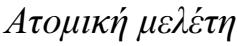

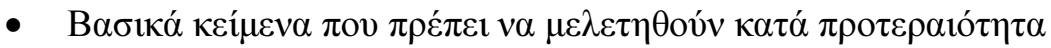

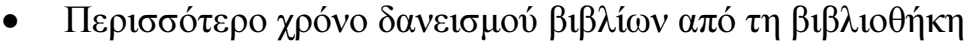

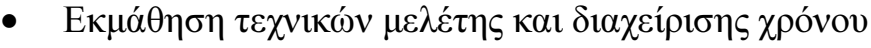

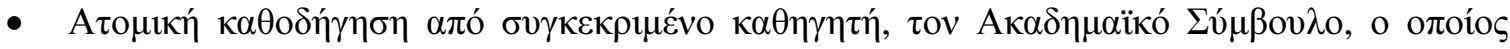

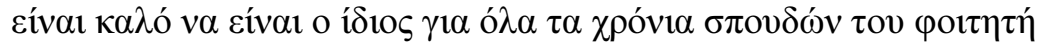

$E \xi \varepsilon \tau \alpha ́ \sigma \varepsilon l \varsigma$

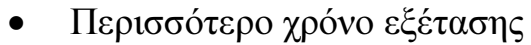

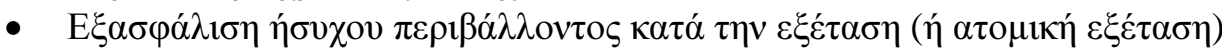

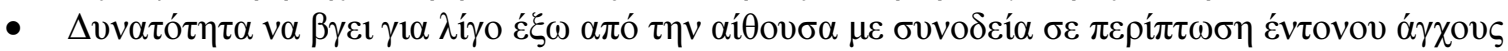

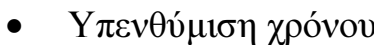

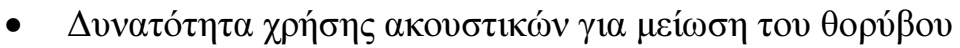

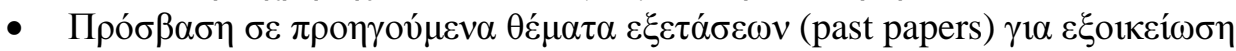

- $\Theta \varepsilon ́ \mu \alpha \tau \alpha \alpha \pi \lambda \alpha ́, \sigma \alpha \varphi \eta ́ ~ \kappa \alpha l ~ \kappa \alpha \tau \alpha v o \eta \tau \alpha ́$

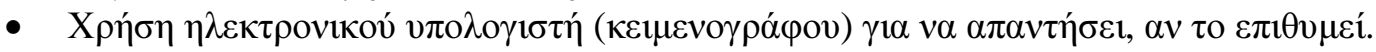

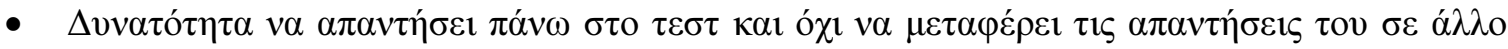

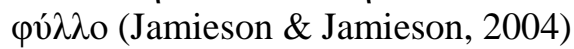

$\Gamma \rho \alpha \pi \tau \varepsilon ́ \varsigma \varepsilon \rho \gamma \alpha \sigma i \varepsilon \varsigma$

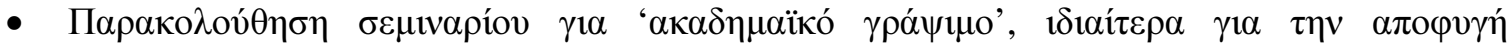

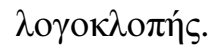

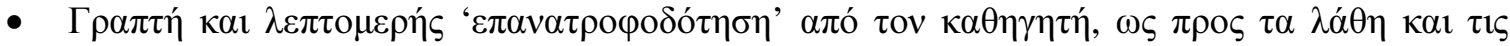

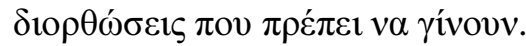

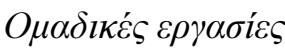

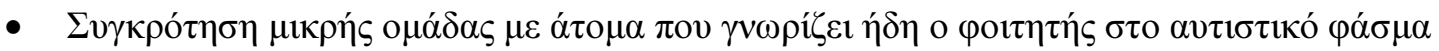

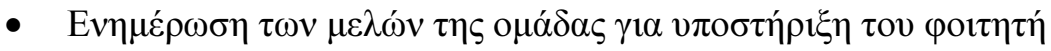

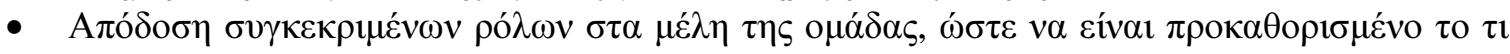

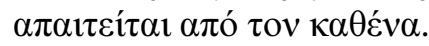

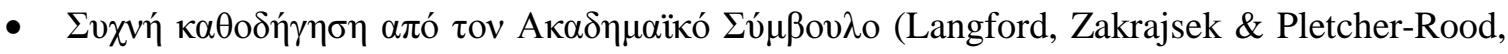
2008).

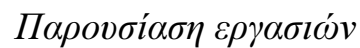

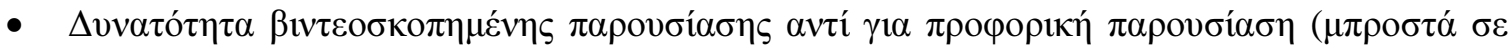
кotvó) 


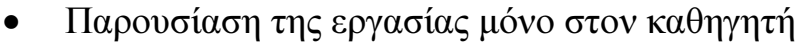

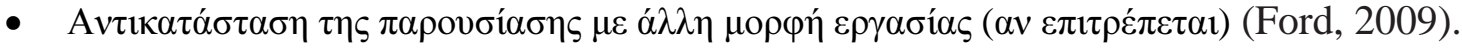

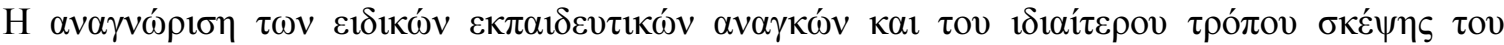

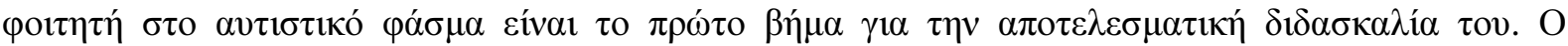

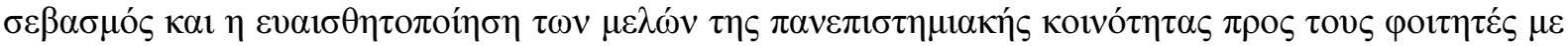

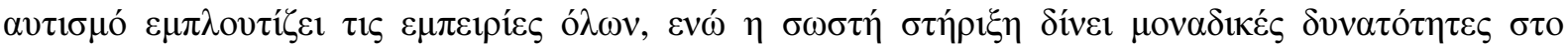

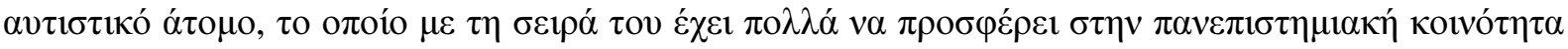
(Howley, $\chi \cdot \eta$ ).

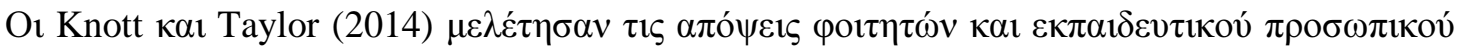

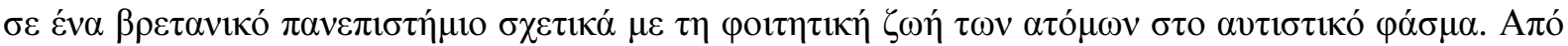

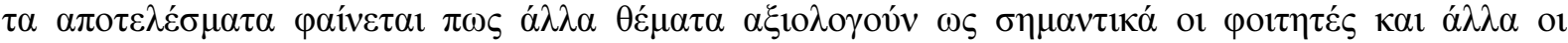

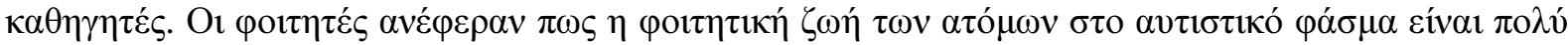

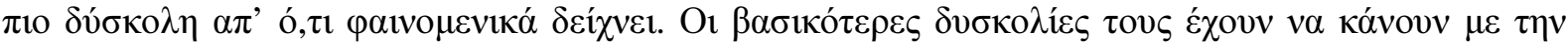

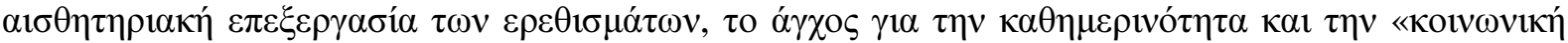

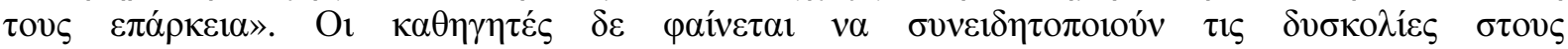

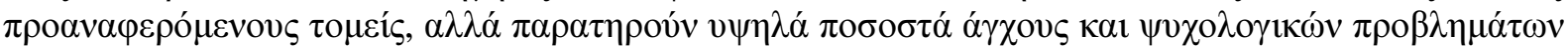

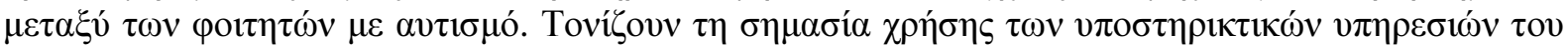

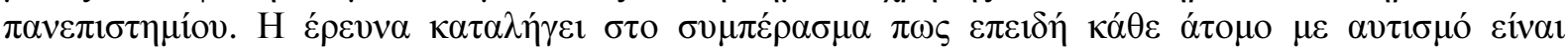

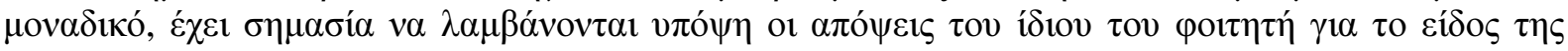

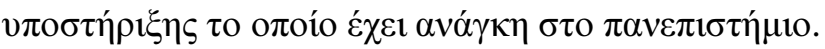

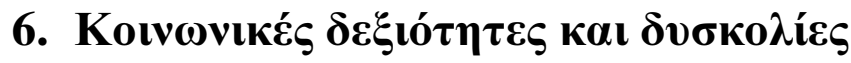

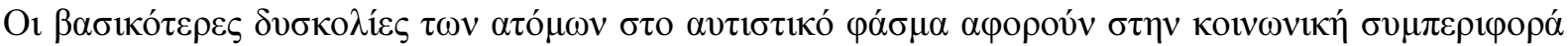

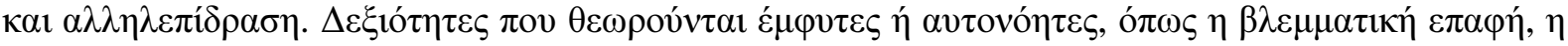

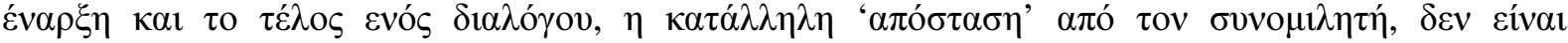

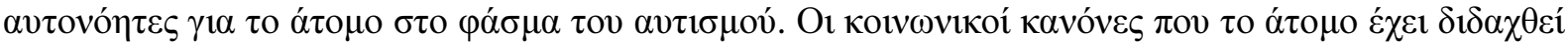

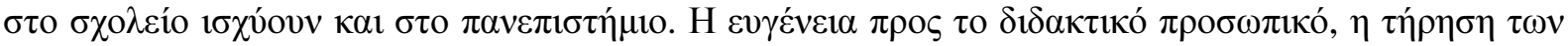

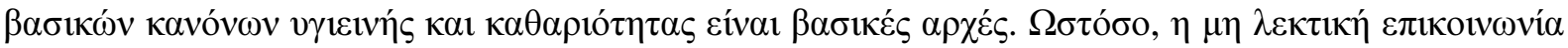

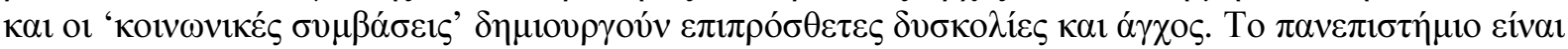

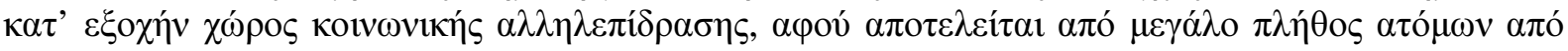

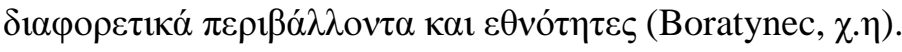

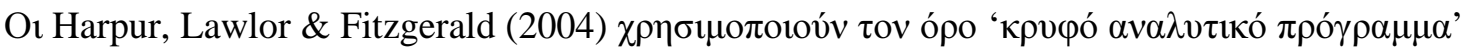

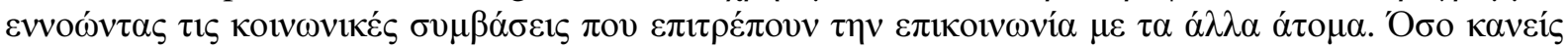

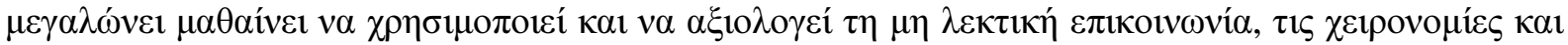

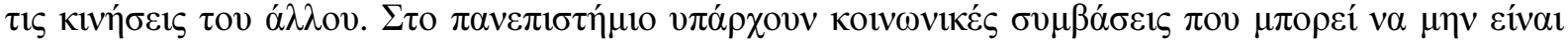

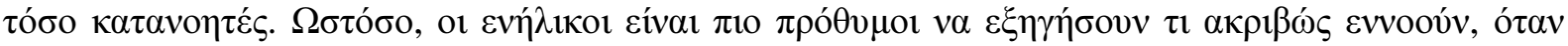

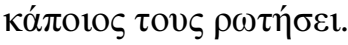

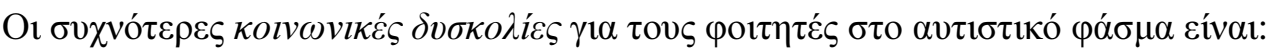

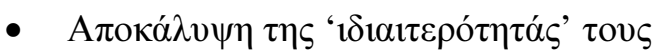

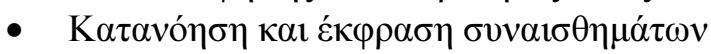

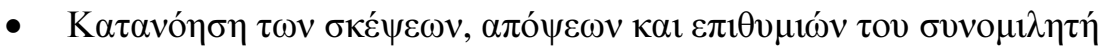

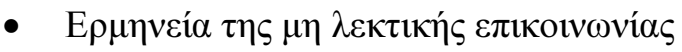

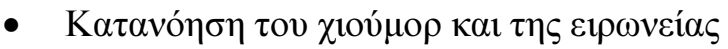

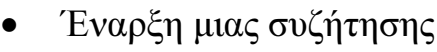

- $\Sigma v \mu \mu \varepsilon \tau о \chi \eta ́ ~ \sigma \varepsilon \mu 1 \alpha \sigma v \zeta \eta \dot{\tau} \eta \sigma \eta$

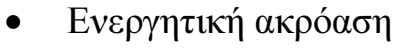




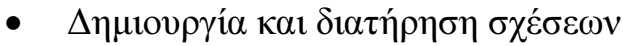

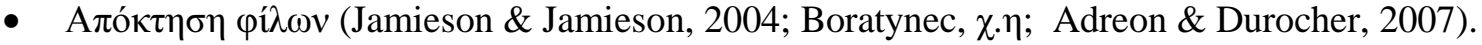

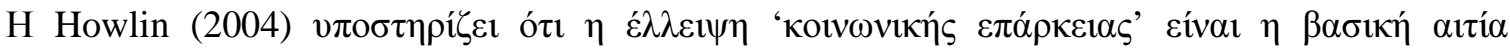

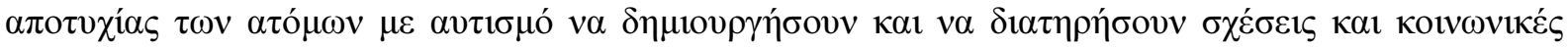

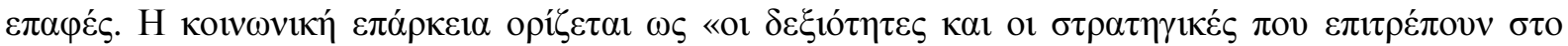

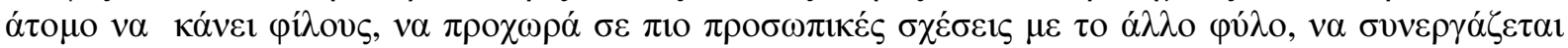

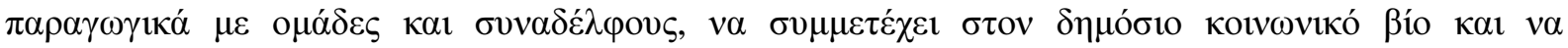

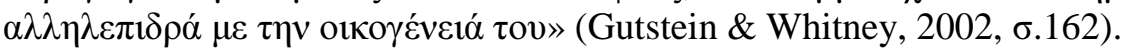

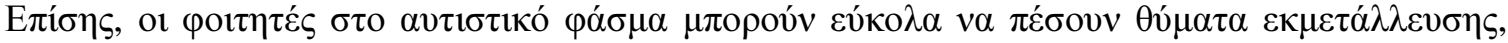

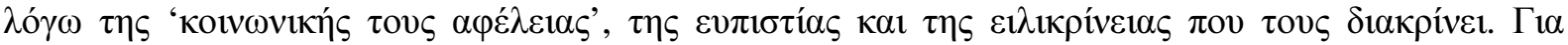

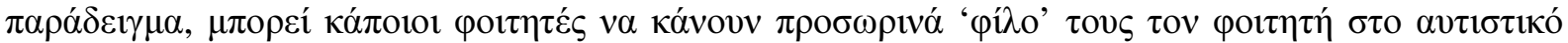

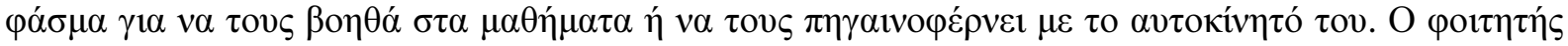

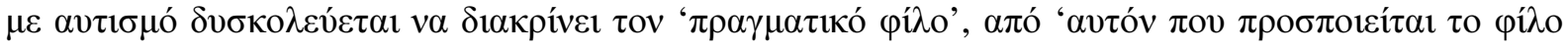

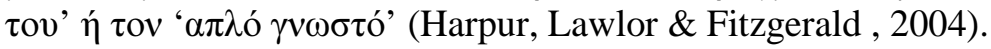

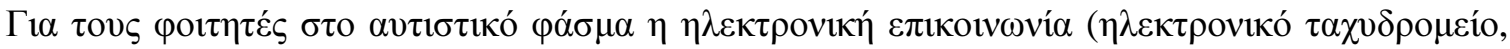

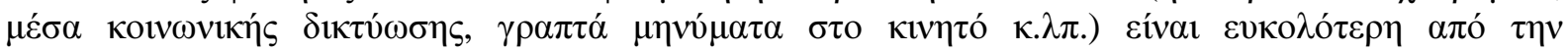

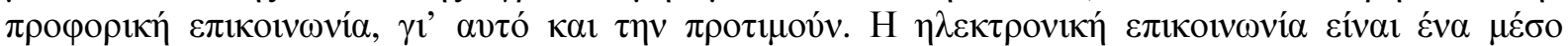

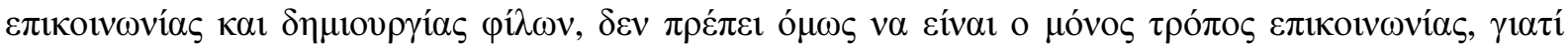

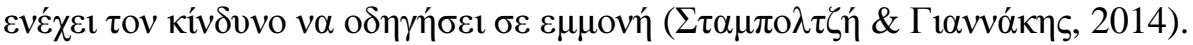

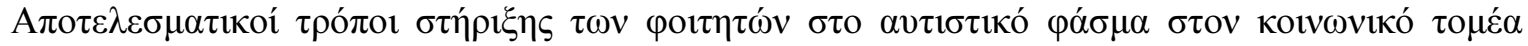
Eíval:

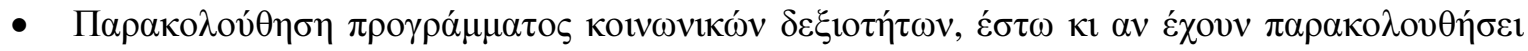

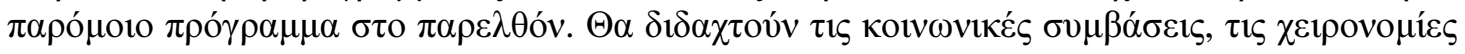

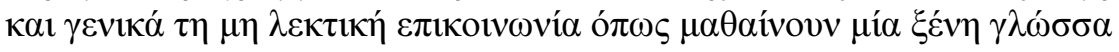

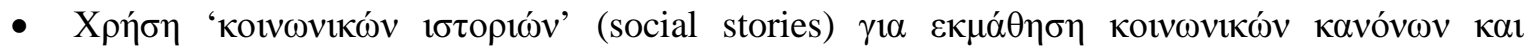

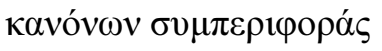

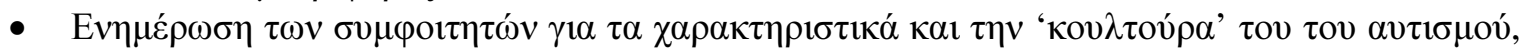

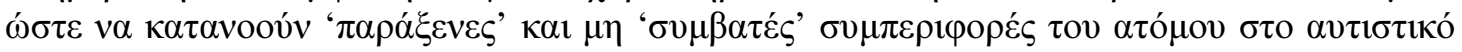
$\varphi \alpha ́ \sigma \mu \alpha$ (Howley, $\chi . \eta$ )

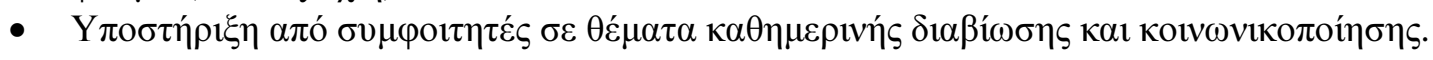

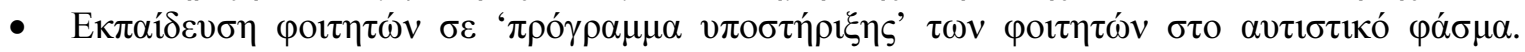

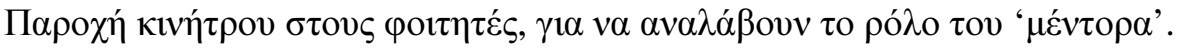

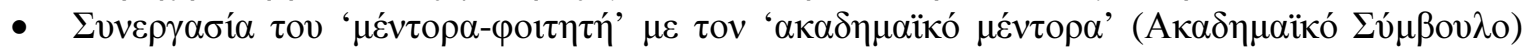

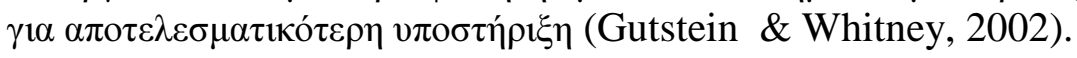

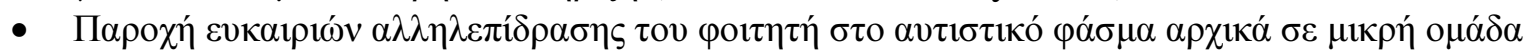

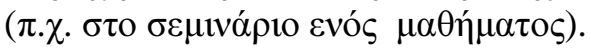

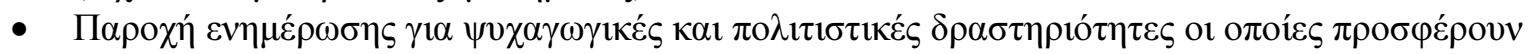

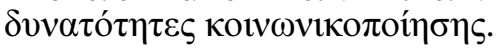

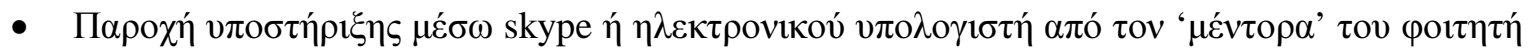

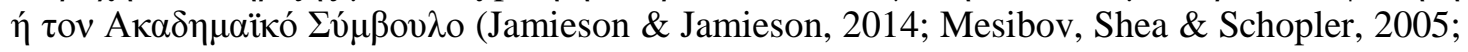
Hastwell et al., 2013).

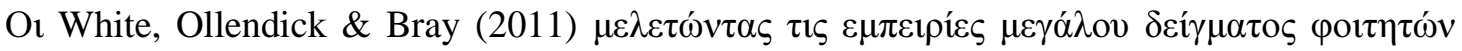

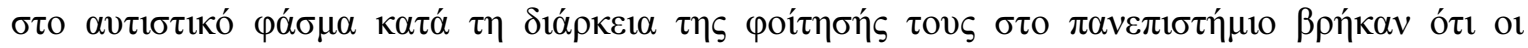

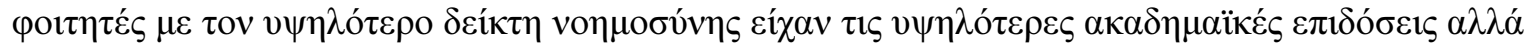

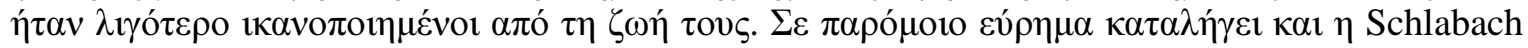

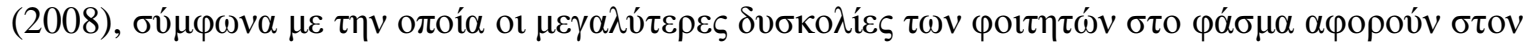

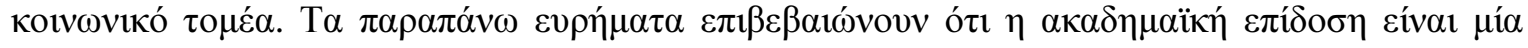




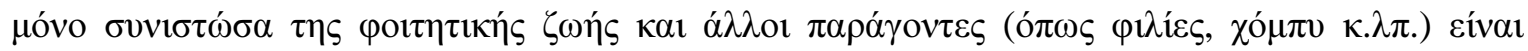

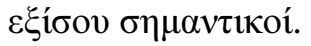

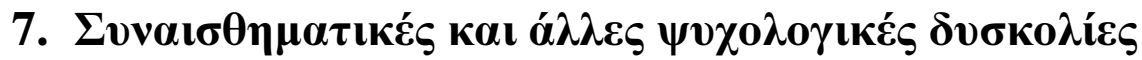

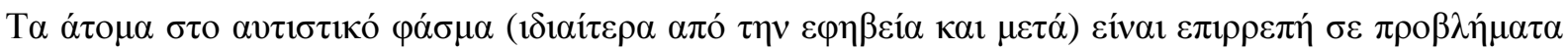

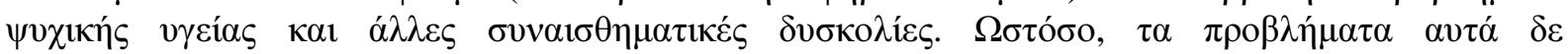

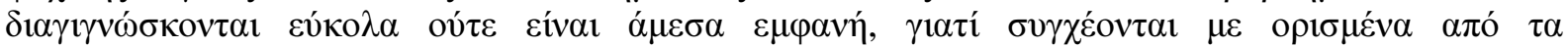

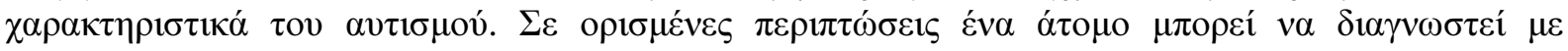

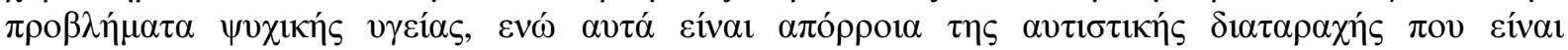

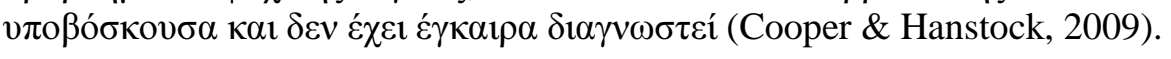

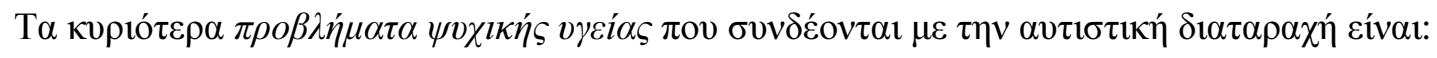

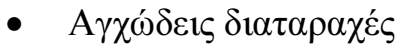

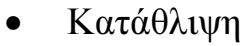

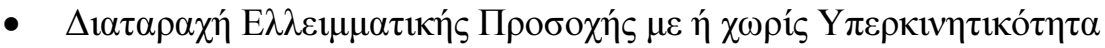

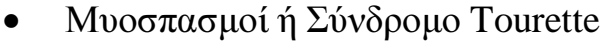

- $\Delta 1 \alpha \tau \alpha \rho \alpha \chi \varepsilon ́ \varsigma \pi \rho o ́ \sigma \lambda \eta \psi \eta \varsigma \tau \rho о \varphi \eta ́ s$

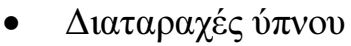

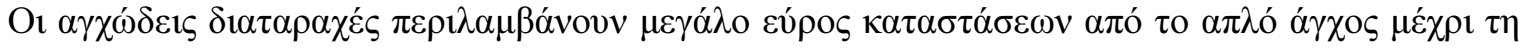

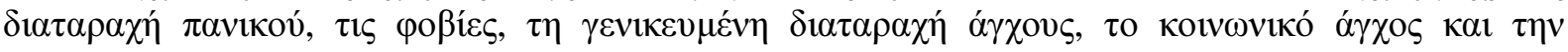

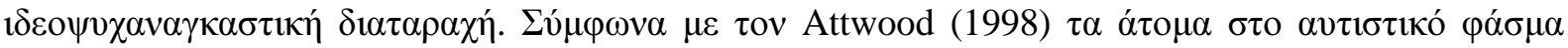

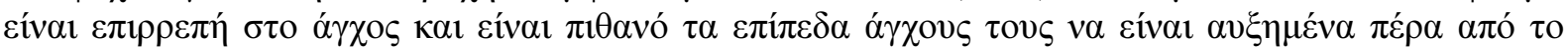

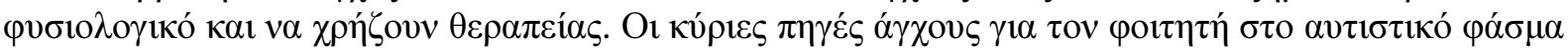

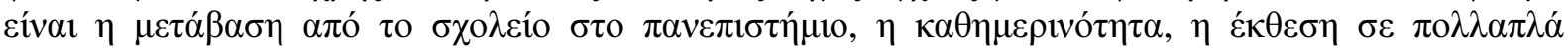

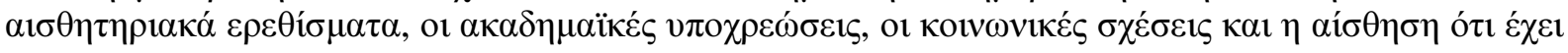

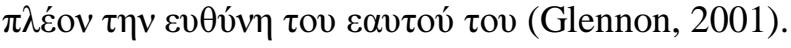

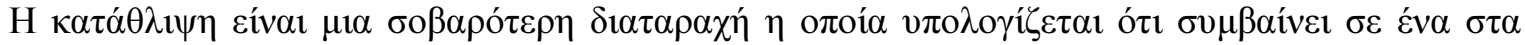

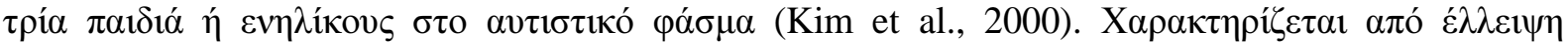

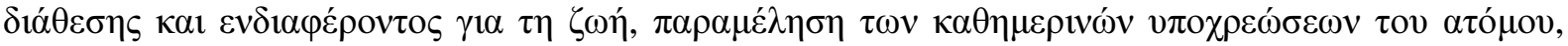

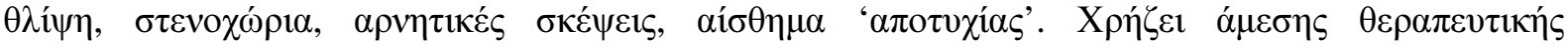

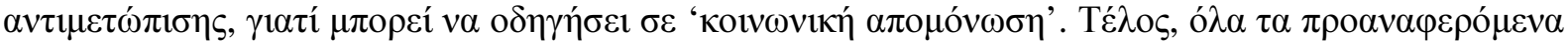

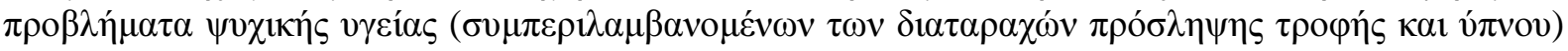

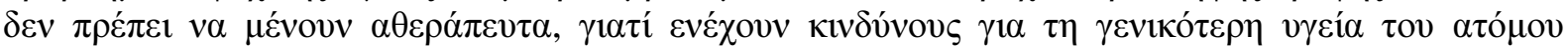

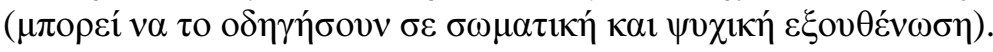

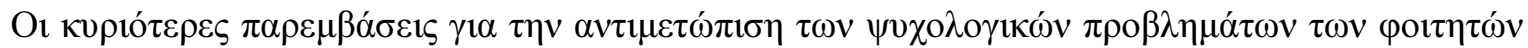

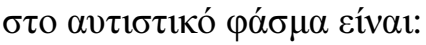

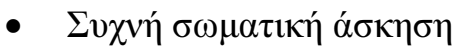

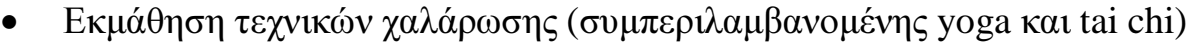

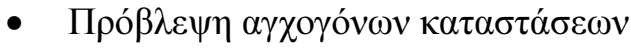

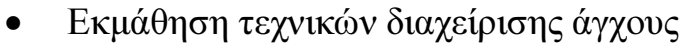

- Ev $\alpha \sigma \chi \delta ́ \lambda \eta \sigma \eta \mu \varepsilon \chi o ́ \mu \pi v$

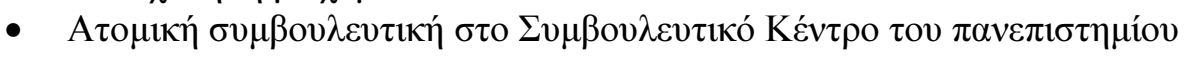

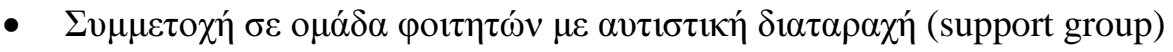

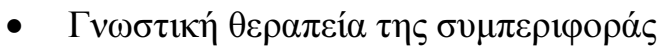

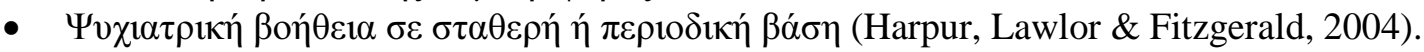




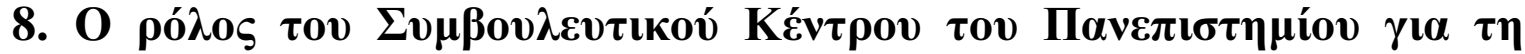

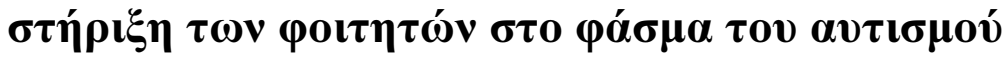

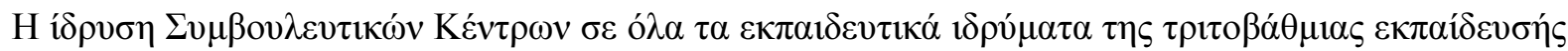

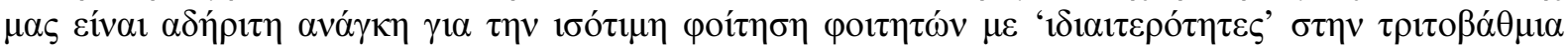

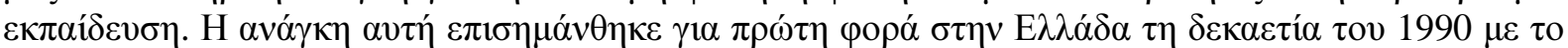

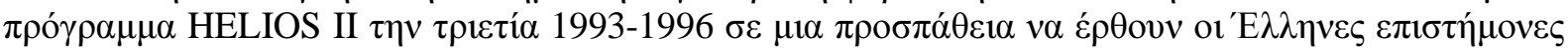

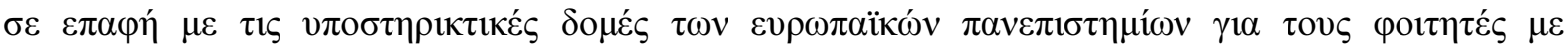

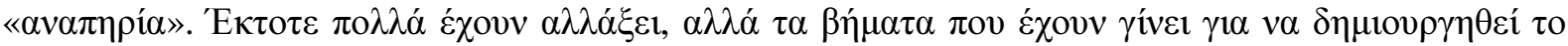

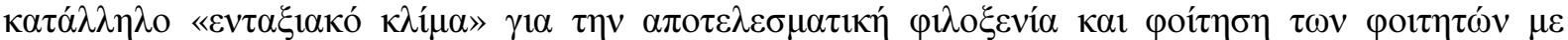

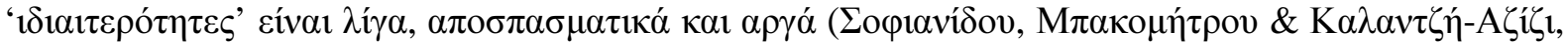
2005).

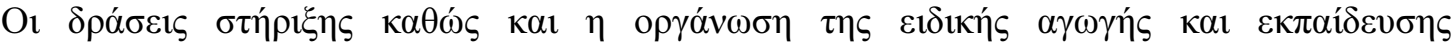

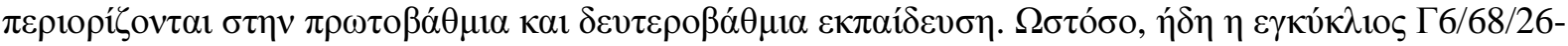

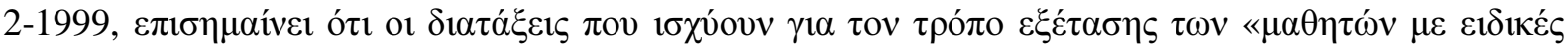

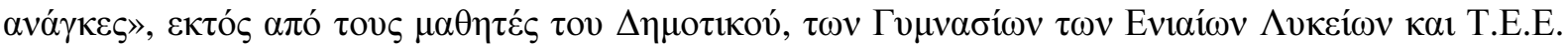

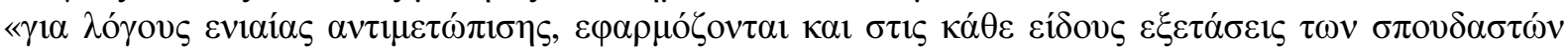

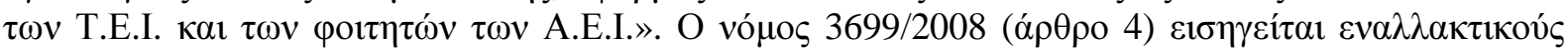

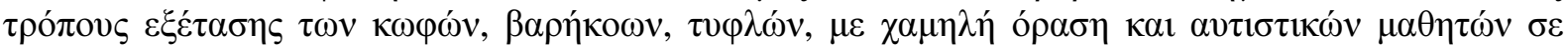

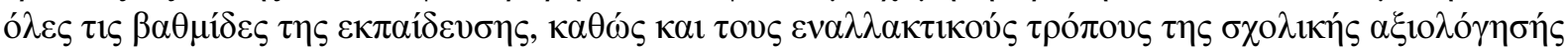

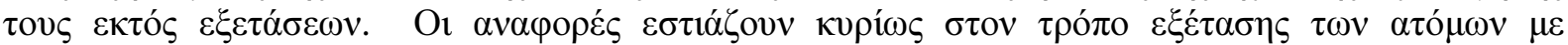

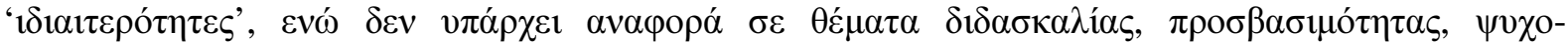

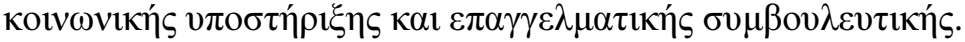

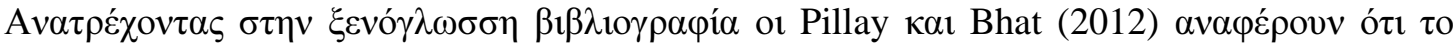

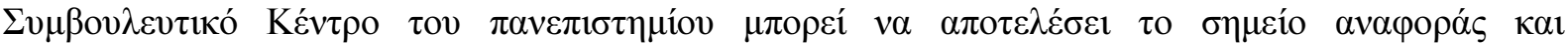

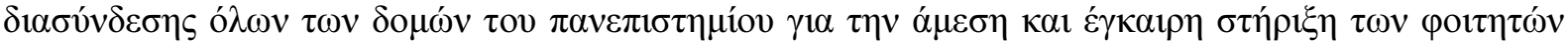

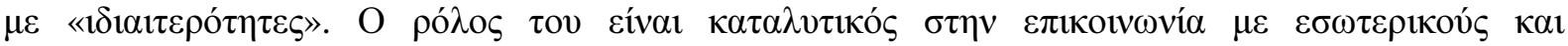

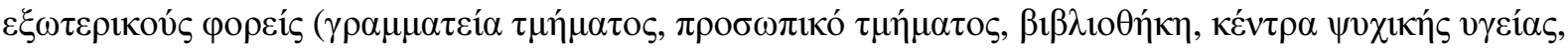

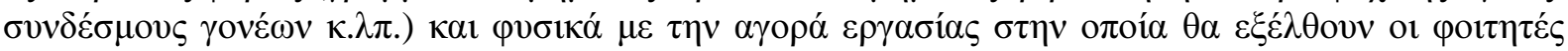

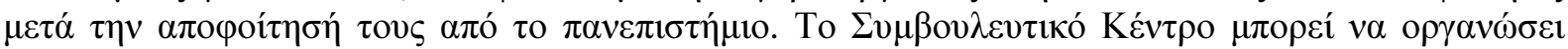

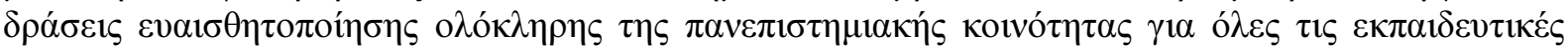

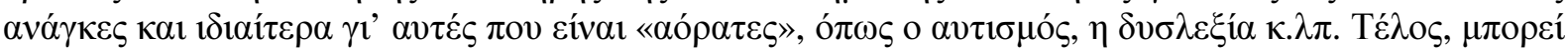

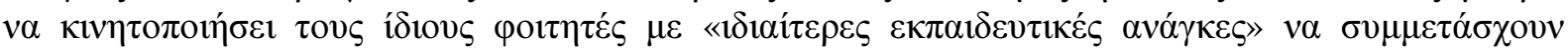

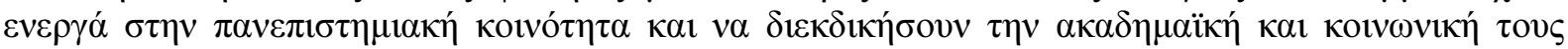
$\varepsilon \dot{v} \tau \alpha \xi \eta$.

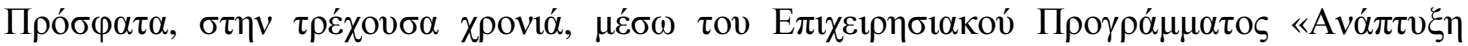

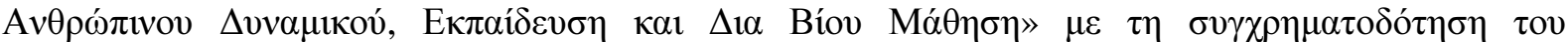

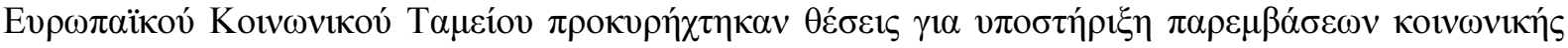

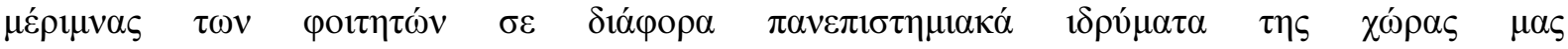

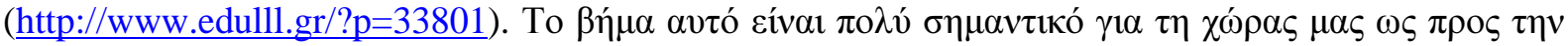

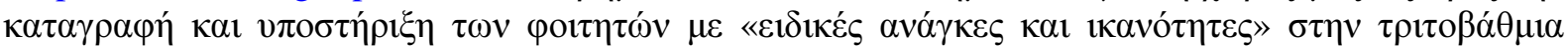
$\varepsilon \kappa \pi \alpha i \delta \varepsilon v \sigma \eta$.

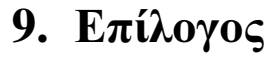

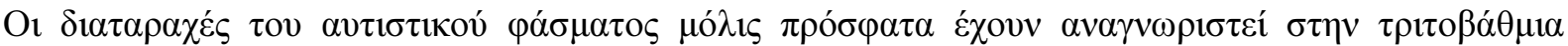

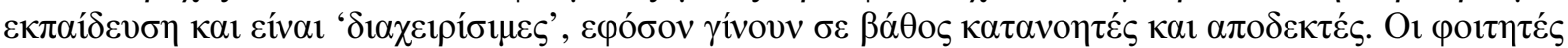

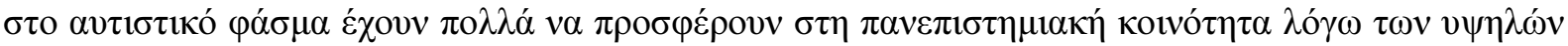

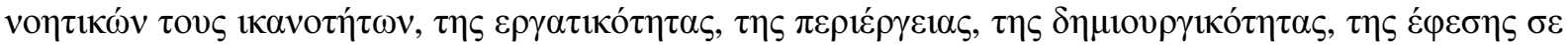

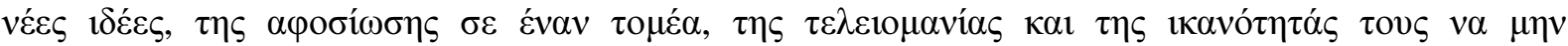

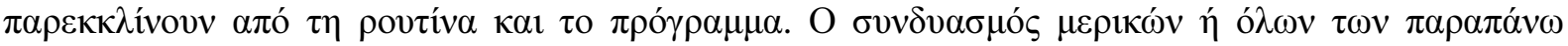




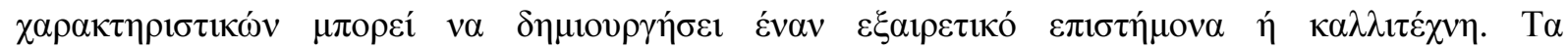

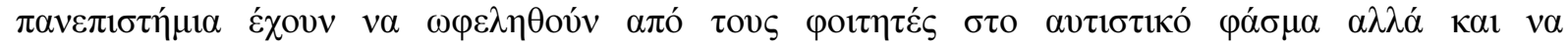

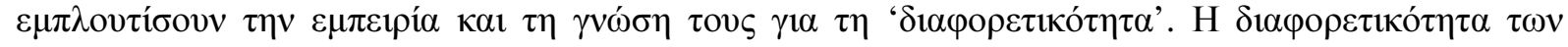

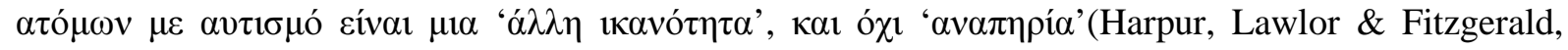
2004).

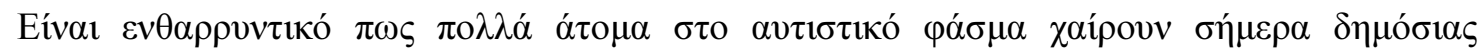

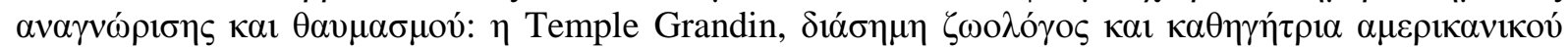

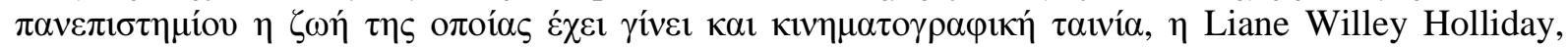

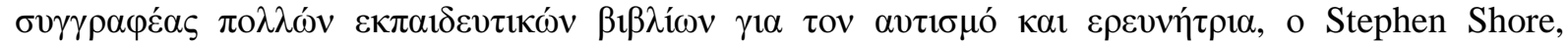

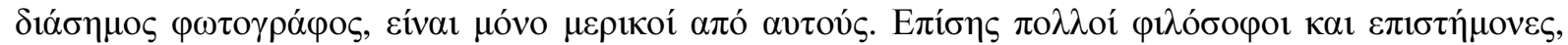

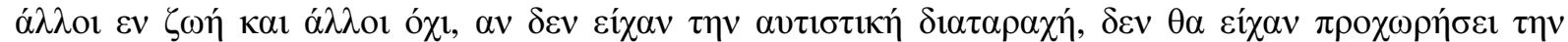

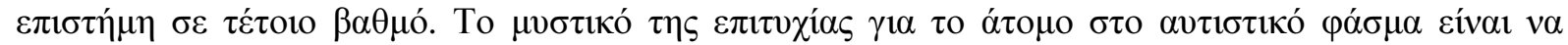

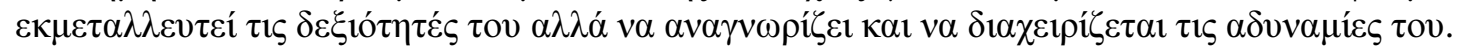

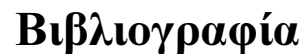

Adreon, D. \& Durocher, S. (2007). Evaluating the college transition needs of individuals with high functioning autism disorders. Intervention in School and Clinic, 42 (5), 271-279. DOI: $10.1177 / 10534512070420050201$

American Psychiatric Association. (2000). Diagnostic and statistical manual of mental disorders: $D S M-I V-T R$ (4th ed., text revision). Washington, DC: Author.

American Psychiatric Association. (2013). Diagnostic and statistical manual of mental disorders (5th ed.). Washington, DC: Author.

Attwood, T. (1998). Asperger's syndrome. London: Jessica Kingsley.

Attwood, T. (2007). Relationship problems of adults with Asperger's syndrome. Good Autism Practice 8, 13-19.

Baird, G., Simonoff, E., Pickles, A., Chandler, S., Loucas,T., Meldrum, D. \& Charman,T. (2006). Prevalence of disorders of the autism spectrum in a population cohort of children in South Thames: the Special Needs and Autism Project (SNAP). Lancet, 368, 210-215.

Baron-Cohen, S., Scott, F., Allison, C., Williams, J., Bolton, P., Matthews, F. \& Brayne, C. (2009). Prevalence of autism spectrum conditions: UK school-based population study. The British Journal of Psychiatry, 194, 500-509. doi: 10.1192/bjp.bp.108.059345

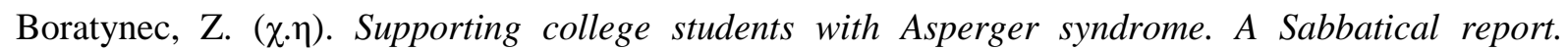
Counselor, Special Services: Saddleback College.

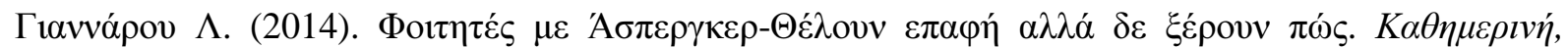

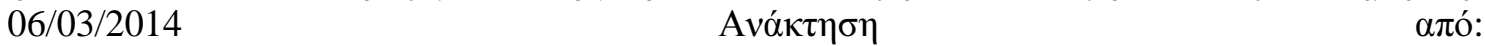

http://www.kathimerini.gr/756748/article/epikairothta/episthmh/foithtes-me-aspergker---

8eloyn-epafh-alla-den-3eroyn-pws.

Center for Disease Control (2007). CDC releases new data on Autism Spectrum. Disorders (HFA OR

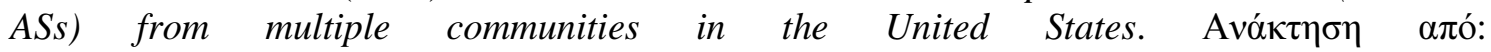
http://www.cdc.gov/od/oc/media/pressrel/2007/r070208.htm (12/12/2007).

Center for Disease Control (2014). Autism and Developmental Disabilities Monitoring Network Surveillance Year 2010 Principal Investigators. Prevalence of autism spectrum disorder among 
children aged 8 years- Autism and Developmental Disabilities Monitoring Network, 11 sites, United States, 2010. MMWR Surveillance Summaries 2014; 63(No. SS-2). Atlanta, GA: Centers for Disease Control and Prevention.

Cooper, K.L \& Hanstock, T.L. (2009). Confusion between depression and autism in a highfunctioning child. Clinical case studies, 8(1), 59-71._doi:10.1177/1534650108327012

Digest of Education Statistics (2009). Table 240. Number and percentage distribution of students enrolled in postsecondary institutions, by level, disability status, and selected student

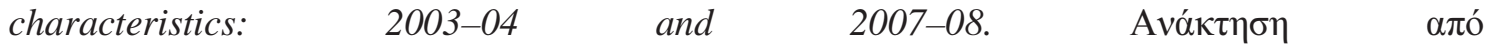
http://nces.ed.gov/programs/digest/d10/tables/dt10_240.asp (04/10/2013).

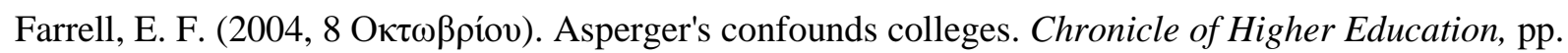
A35-A36.

Filler, C. \& Rosenhein, M. (2012). IEP transition components: Transition to adulthood. Guidelines for

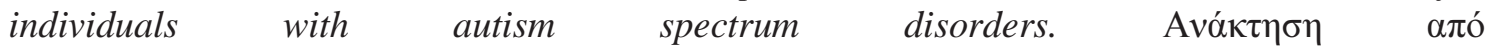
http://www.ocali.org/up_doc/TG12_IEPComponents.pdf

Ford, C.D. (2009). An investigation of support programs for college students with high functioning autism or Asperger syndrome. Unpublished doctoral dissertation. University of MissouriColumbia.

Glenon, T.J. (2001). The stress of the university experience for students with Asperger syndrome. Work, 17, 183-190

Grant, R. \& Nozyce, M. (2013). Proposed changes to the American Psychiatric Association diagnostic criteria for autism spectrum disorder: Implications for young children and their families. Maternal Child Health Journal, 17, 586-592. DOI 10.1007/s10995-013-1250-9

Gutstein, S. E., \& Whitney, T. (2002). Asperger Syndrome and the development of social competence. Focus on Autism \& Other Developmental Disabilities, 17(3), 161-172. doi:10.1177/10883576020170030701

Harrison, J. \& Hare, D.J. (2004). Brief report: assessment of sensory abnormalities in people with autistic spectrum disorders. Journal of Autism and Developmental Disorders, 34 (6), 727-730.

Harpur, J., Lawlor, M \& Fitzgerald, M. (2004). Succeeding at college with Asperger syndrome. London: Jessica Kingsley.

Hastwell, J., Harding, J., Martin, N. \& Baron-Cohen, S. (2013). Asperger syndrome student project, 2009-12 (Final project report). Disability Resource Centre: University of Cambridge.

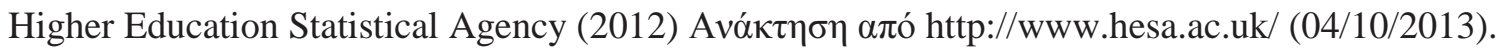

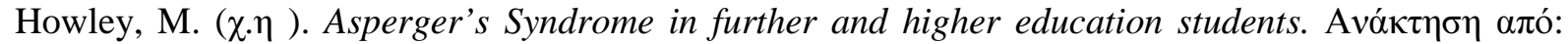
http://www.brainhe.com $(02 / 10 / 2013)$

Howlin, P. (2004) Autism and Asperger Syndrome: preparing for adulthood. (2 ${ }^{\text {nd }}$ ed.). London: Routledge.

Hughes, C., Golas, M., Cosgriff, J., Brigham, N., Edwards, C., \& Cashen, K. (2011). Effects of a social skills intervention among high school students with intellectual disabilities and autism 
and their general education peers. Research and Practice for Persons with Severe Disabilities, 36 (1), 46-61. doi: 10.2511/rpsd.36.1-2.46

Hurst, D. \& Smerdon, B. (2000). Postsecondary students with disabilities: Enrollment, services, and persistence. Education Statistics Quarterly, 2 (3), 55-58.

Jabr, F. (2012). Redefining Autism: Will New DSM-5 Criteria for ASD Exclude Some People?

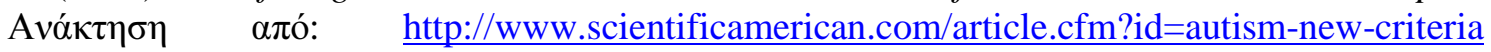
(20/10/2014).

Jamieson, J. \& Jamieson, C. (2004). Managing Asperger syndrome at college and university: A resource for students, tutors and support services. London: David Fulton Publishers.

Kim, J. A., Szatmari, P., Bryson, S. E., Streiner, D. L. \& Wilson, F. (2000). The prevalence of anxiety and mood problems among children with autism and Asperger Syndrome. Autism, 4 (2), 117 132. doi: $10.1177 / 1362361300004002002$

Knott, F. \& Taylor, A. (2014). Life at university with Asperger syndrome:a comparison of student and staff perspectives. International Journal of Inclusive Education, 18 (4), 411-426, DOI: $10.1080 / 13603116.2013 .781236$

Langford, S.J.,Zakrajsek, T. \& Pletcher-Rood, S. (2008). Teaching students with Asperger syndrome (and other disabilities) in the college classroom: Creating an inclusive learning environment. Journal on Excellence in College Teaching, 19 (2-3), 107-133.

Lawrence, D. H., Alleckson, D.A. \& Bjorklund, P. (2010). Beyond the roadblocks: Transitioning to adulthood with Asperger's syndrome. Archives of Psychiatric Nursing, 24(4), 227-238. DOI: http://dx.doi.org/10.1016/j.apnu.2009.07.004

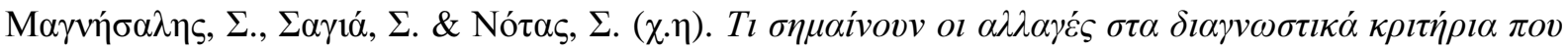

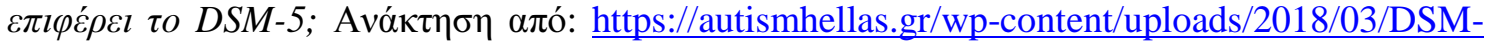
$\mathrm{V} \% \mathrm{CE} \% 95 \% \mathrm{CE} \% \mathrm{~A} 1 \% \mathrm{CE} \% \mathrm{~A} 9 \% \mathrm{CE} \% \mathrm{~A} 4 \% \mathrm{CE} \% 97 \% \mathrm{CE} \% \mathrm{~A} 3 \% \mathrm{CE} \% 95 \% \mathrm{CE} \% 99 \% \mathrm{CE} \% \mathrm{~A} 3 . \mathrm{p}$ df $(05 / 05 / 2017)$

Mesibov, G B, Shea, V and Schopler, E (2005) The TEACCH Approach to Autism Spectrum Disorders. New York: Plenum Press.

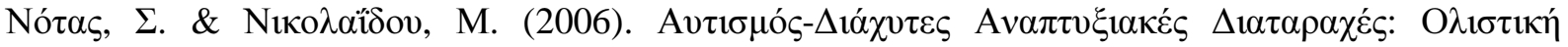

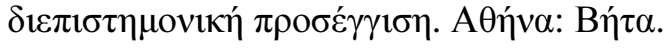

Özerk (2016). The issue of prevalence of autism/ASD. International Electronic Journal of Elementary Education, 9(2), 263-306.

Pillay, Y. \& Suniti Bhat, C. (2012) Facilitating support for students with Asperger's Syndrome. Journal of College Student Psychotherapy, 26 (2), 140-154. DOI: 10.1080/87568225.2012.659161

Schlabach, T. (2008). The college experience of students with Asperger's disorder: Perceptions of the students themselves and of college disability service providers who work with these students. Unpublished doctoral dissertation. Illinois State University.

Smith, C. (2007). Support services for students with Asperger's syndrome in higher education. College Student Journal, 41(3), 515-531. 


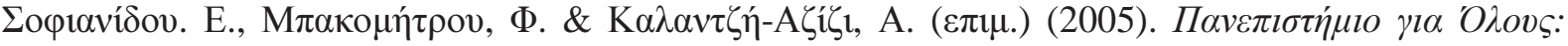

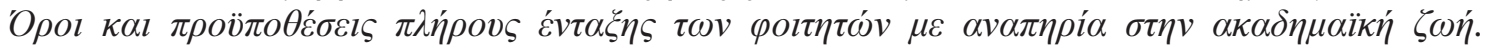

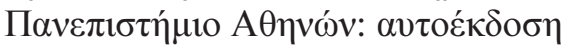

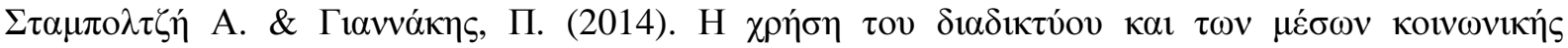

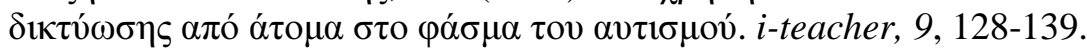

Swedo, S. E. (2012). An update on the DSM-5 recommendations for autism spectrum disorder and

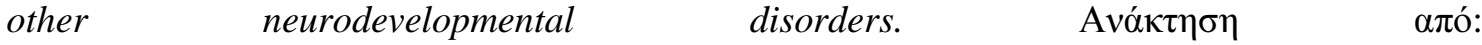
http://iacc.hhs.gov/events/2012/slides susan_swedo 071012.pdf (07/02/2013).

Taylor, M.J. (2005). Teaching students with autistic spectrum disorders in HE. Education \& Training, 47 (7), 484-495. http://dx.doi.org/10.1108/00400910510626330

Test, D., Mazzoti, V., Mustian, A., Fowler, C., Kortering, L. \& Kohler, P. (2009). Evidence-based practices in secondary transition. Career Development and Transition For Exceptional Individuals, 32(2), 115-128. doi: 10.1177/0885728809336859

Thomas, J., \& Harden, A. (2008). Methods for the thematic synthesis of qualitative research in systematic reviews. BMC Medical Research Methodology, 8, 45. doi: 10.1186/1471-2288-8-45.

Tsai, L. Y. (2003). Pervasive developmental disorders. Briefing paper. National Dissemination Center

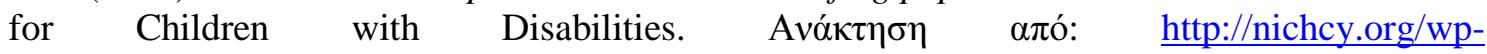
content/uploads/docs/fs20.pdf (11/10/2012)

Wehman, P. (2006). Life beyond the classroom: Transition strategies for young people with disabilities ( $4^{\text {th }}$ ed.). Baltimore: Paul Brooks Publishing Co.

White, S.W., Ollendick, T.H. \& Bray, B.C (2011). College students on the autism spectrum: prevalence and associated problems. Autism, 15 (6), 683-701. doi: 10.1177/1362361310393363

Wolf, L. E., Thierfeld Brown, J., \& Kukiela Bork, G. R. (2009). Students with Asperger syndrome: A guide for college personnel. Overland Park, KS: Autism Asperger Publishing Company. 